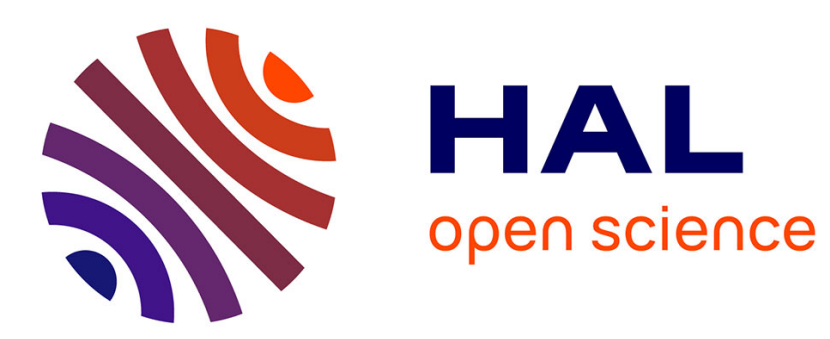

\title{
Spreading Properties and Complex Dynamics for Monostable Reaction-Diffusion Equations
}

\author{
François Hamel, Grégoire Nadin
}

\section{To cite this version:}

François Hamel, Grégoire Nadin. Spreading Properties and Complex Dynamics for Monostable Reaction-Diffusion Equations. Communications in Partial Differential Equations, 2012, 37, pp.511537. 10.1080/03605302.2011.647198 . hal-01257400

\section{HAL Id: hal-01257400 https://hal.science/hal-01257400}

Submitted on 16 Jan 2016

HAL is a multi-disciplinary open access archive for the deposit and dissemination of scientific research documents, whether they are published or not. The documents may come from teaching and research institutions in France or abroad, or from public or private research centers.
L'archive ouverte pluridisciplinaire HAL, est destinée au dépôt et à la diffusion de documents scientifiques de niveau recherche, publiés ou non, émanant des établissements d'enseignement et de recherche français ou étrangers, des laboratoires publics ou privés. 


\title{
Spreading properties and complex dynamics for monostable reaction-diffusion equations
}

\author{
François Hamel ${ }^{\mathrm{a}}$ and Grégoire Nadin ${ }^{\mathrm{b} *}$ \\ a Aix-Marseille Université \& Institut Universitaire de France \\ LATP, UMR 6632, Avenue Escadrille Normandie-Niemen, F-13397 Marseille Cedex 20, France \\ b CNRS, UMR 7598, Laboratoire Jacques-Louis Lions, F-75005 Paris, France
}

\begin{abstract}
This paper is concerned with the study of the large-time behaviour of the solutions $u$ of a class of one-dimensional reaction-diffusion equations with monostable reaction terms $f$, including in particular the classical Fisher-KPP nonlinearities. The nonnegative initial data $u_{0}(x)$ are chiefly assumed to be exponentially bounded as $x$ tends to $+\infty$ and separated away from the unstable steady state 0 as $x$ tends to $-\infty$. On the one hand, we give some conditions on $u_{0}$ which guarantee that, for some $\lambda>0$, the quantity $c_{\lambda}=\lambda+f^{\prime}(0) / \lambda$ is the asymptotic spreading speed, in the sense that $\lim _{t \rightarrow+\infty} u(t, c t)=1$ (the stable steady state) if $c<c_{\lambda}$ and $\lim _{t \rightarrow+\infty} u(t, c t)=0$ if $c>c_{\lambda}$. These conditions are fulfilled in particular when $u_{0}(x) e^{\lambda x}$ is asymptotically periodic as $x \rightarrow+\infty$. On the other hand, we also construct examples where the spreading speed is not uniquely determined. Namely, we show the existence of classes of initial conditions $u_{0}$ for which the $\omega$-limit set of $u(t, c t+x)$ as $t$ tends to $+\infty$ is equal to the whole interval $[0,1]$ for all $x \in \mathbb{R}$ and for all speeds $c$ belonging to a given interval $\left(\gamma_{1}, \gamma_{2}\right)$ with large enough $\gamma_{1}<\gamma_{2} \leq+\infty$.
\end{abstract}

\section{Introduction and notion of spreading speeds}

We study in this paper the large-time behaviour of the solutions of monostable reactiondiffusion equations of the type

$$
\begin{cases}\partial_{t} u-\partial_{x x} u=f(u), & t>0, x \in \mathbb{R} \\ u(0, x)=u_{0}(x) & \text { for a.e. } x \in \mathbb{R}\end{cases}
$$

where the reaction term $f:[0,1] \rightarrow \mathbb{R}$ is a $\mathcal{C}^{1}$ function such that

$$
f(0)=f(1)=0, f(s)>0 \text { if } s \in(0,1), f^{\prime}(0)>0,
$$

${ }^{*}$ The first author is indebted to the Alexander von Humboldt Foundation for its support. Both authors are also supported by the French "Agence Nationale de la Recherche" within the project PREFERED. 
and $u_{0}$ is a measurable initial datum such that $u_{0} \not \equiv 0, u_{0} \not \equiv 1$ and $0 \leq u_{0}(x) \leq 1$ for almost every $x \in \mathbb{R}$ (the quantity $u$ typically stands for a normalized density in the applications in population dynamics models). Under these hypotheses, the Cauchy problem (1.1) is well-posed, the solution $u$ is classical for $t>0$, and $u(t, x) \in(0,1)$ for all $t>0, x \in \mathbb{R}$.

Such equations have first been investigated by Fisher [7] and Kolmogorov, Petrovski and Piskunov [12]. Among other results, these authors proved that, when $f(s)=s(1-s)$ and $u_{0}$ is the Heaviside function, that is $u_{0}=1$ on $(-\infty, 0)$ and 0 on $(0,+\infty)$, then

$$
\begin{cases}\min _{x \leq c t} u(t, x) \rightarrow 1 \text { as } t \rightarrow+\infty & \text { if } c<c^{*}, \\ \max _{x \geq c t} u(t, x) \rightarrow 0 \text { as } t \rightarrow+\infty & \text { if } c>c^{*},\end{cases}
$$

with $c^{*}=2$ in this case. Such properties are called spreading properties and the quantity $c^{*}$ is called the spreading speed associated with the initial datum $u_{0}$. This result has been extended by Aronson and Weinberger [1] to multidimensional media and positive nonlinearities satisfying (1.2). In particular, it is proved in [1] that, in dimension 1, formula (1.3) still holds when $u_{0}$ is the Heaviside function, for some positive real number $c^{*}$ which only depends on $f$.

For general functions $f$ satisfying (1.2), this threshold $c^{*}$ also turns out to be the minimal speed of existence of travelling fronts solutions of equation (1.1). Namely, we say that a solution $u$ of (1.1) is a travelling front if it can be written as $u(t, x)=U_{c}(x-c t)$, with $U_{c}(-\infty)=1, U_{c}(+\infty)=0$ and $0<U_{c}<1$ in $\mathbb{R}$. In this case, we say that $c$ is the speed of the travelling front solution $u$. It is well known $[1,8]$ that if $f$ satisfies $(1.2)$, then there exists a speed $c^{*}$ such that there exists a travelling front solution of (1.1) with speed $c$ if and only if $c \geq c^{*}$. Furthermore, if $f$ satisfies the now-called Fisher-KPP assumption, that is if

$$
0<f(s) \leq f^{\prime}(0) s \text { for all } s \in(0,1)
$$

then $c^{*}=2 \sqrt{f^{\prime}(0)}$. For general functions $f$ satisfying $(1.2)$, one has $c^{*} \geq 2 \sqrt{f^{\prime}(0)}$, see $[1,8]$. Lastly, for each $c \geq c^{*}$, the profile $U_{c}$ associated with the travelling front of speed $c$ is decreasing on $\mathbb{R}$ and unique up to translation and, if $c>c^{*}$, there exists $M>0$ such that

$$
U_{c}(z) \sim M e^{-\lambda z} \text { as } z \rightarrow+\infty
$$

where

$$
\lambda=\frac{c-\sqrt{c^{2}-4 f^{\prime}(0)}}{2}
$$

is the smallest root of the equation $\lambda^{2}-\lambda c+f^{\prime}(0)=0$. Thus, the estimates of the spreading speeds, as defined below, are expected to be given in terms of the exponential decay rate of the initial condition.

Before stating our main results in the next section, we define in this section the notions of minimal and maximal spreading speeds for the solutions $u$ of (1.1), with a class of initial conditions $u_{0}: \mathbb{R} \rightarrow[0,1]$ which are much more general than the Heaviside function.

Definition 1.1 We say that a function $u_{0} \in L^{\infty}(\mathbb{R})$ is front-like if $0 \leq u_{0}(x) \leq 1$ for a.e. $x \in \mathbb{R}$ and there exist $x_{-} \in \mathbb{R}$ and $\delta>0$ such that

$$
u_{0}(x) \geq \delta \text { for a.e. } x<x_{-} \text {and } \lim _{x \rightarrow+\infty}\left\|u_{0}\right\|_{L^{\infty}(x,+\infty)}=0 \text {. }
$$


The term front-like refers to the fact that the values of $u_{0}(x)$ as $x \rightarrow \pm \infty$ are strictly ordered, although the front-like initial $u_{0}$ may not be nonincreasing on $\mathbb{R}$ even up to a set of zero measure. However, these very mild conditions still guarantee that $u(t, x) \rightarrow 0$ as $x \rightarrow+\infty$ for every $t>0$, from standard parabolic estimates and the maximum principle. For such initial data, we still expect the solutions of the Cauchy problem (1.1) to spread, that is the stable state 1 to invade the unstable steady state 0 . At first glance, we could think that a property like (1.3) still holds, where $c^{*}$ would in general be replaced with a quantity $w>0$ which would depend on $u_{0}$. A natural question would then be to compute the speed $w$ of this invasion. In fact, it turns out that some complex dynamics may occur in general. The mild conditions in Definition 1.1 give rise to a large variety of propagation phenomena at large time, some of them being of a completely new type. Thus, in order to quantify the spreading, we are led to introduce two natural quantities: the minimal and the maximal spreading speeds.

Definition 1.2 For a given front-like function $u_{0}$, we define the minimal and maximal spreading speeds $w_{*}\left(u_{0}\right)$ and $w^{*}\left(u_{0}\right)$ of the solution $u$ of $(1.1)$ as

$$
\begin{aligned}
& w_{*}\left(u_{0}\right)=\sup \left\{c \in \mathbb{R}, \inf _{x \leq c t} u(t, x) \rightarrow 1 \text { as } t \rightarrow+\infty\right\}, \\
& w^{*}\left(u_{0}\right)=\inf \left\{c \in \mathbb{R}, \sup _{x \geq c t} u(t, x) \rightarrow 0 \text { as } t \rightarrow+\infty\right\} .
\end{aligned}
$$

It immediately follows from Definition 1.2 that, for any given front-like function $u_{0}$, one has

$$
\inf _{x \leq c t} u(t, x) \rightarrow 1 \text { as } t \rightarrow+\infty \text { for all } c<w_{*}\left(u_{0}\right)
$$

and

$$
\sup _{x \geq c t} u(t, x) \rightarrow 0 \text { as } t \rightarrow+\infty \text { for all } c>w^{*}\left(u_{0}\right)
$$

if $w^{*}\left(u_{0}\right)$ is finite. Actually, we will see below that $w_{*}\left(u_{0}\right)$ can never be $-\infty$, but that $w^{*}\left(u_{0}\right)$, and $w_{*}\left(u_{0}\right)$, are sometimes equal to $+\infty$.

Let us now give some general comparisons and a list of standard examples for which these quantities can be explicitly computed. First, when there is a real number $A$ such that $u_{0}(x)=\sigma \in(0,1]$ for a.e. $x<A$ and $u_{0}(x)=0$ for a.e. $x>A$, it is then well known $[1,12]$ that $w_{*}\left(u_{0}\right)=w^{*}\left(u_{0}\right)=c^{*}$, where $c^{*}$ is the minimal speed of existence of travelling fronts solutions. Using this fact and the parabolic maximum principle, as any front-like function is bounded from below by a space shift of the Heaviside function multiplied by some $\sigma \in(0,1]$, we get that

$$
c^{*} \leq w_{*}\left(u_{0}\right) \leq w^{*}\left(u_{0}\right) \leq+\infty
$$

for any front-like initial datum $u_{0}$. In general, the spreading speeds are strictly larger than $c^{*}$. For example, for any speed $c \geq c^{*}$, if $u(t, x)=U_{c}(x-c t)$ is a travelling front solution of speed $c$, then $w_{*}(u(0, \cdot))=w_{*}\left(U_{c}\right)=w^{*}(u(0, \cdot))=w^{*}\left(U_{c}\right)=c$. Set now

$$
\lambda^{*}=\min \left\{\lambda>0, \lambda^{2}-\lambda c^{*}+f^{\prime}(0)=0\right\}=\frac{c^{*}-\sqrt{c^{* 2}-4 f^{\prime}(0)}}{2},
$$

which is a well defined real number since $c^{*} \geq 2 \sqrt{f^{\prime}(0)}$, consider

$$
u_{0}(x)=\min \left(\sigma, \theta e^{-\lambda x}\right) \text { for all } x \in \mathbb{R}
$$


with $\sigma \in(0,1], \theta>0$ and $\lambda \in\left(0, \lambda^{*}\right)$, and define

$$
c_{\lambda}=\lambda+\frac{f^{\prime}(0)}{\lambda} .
$$

When $f$ satisfies $(1.2)$ and $f^{\prime}(s) \leq f^{\prime}(0)$ for all $s \in[0,1]$, it has been proved through probabilistic methods by McKean [16] and through PDE's methods by Kametaka [11] that the solution $u$ of (1.1) satisfies

$$
\sup _{x \in \mathbb{R}}\left|u(t, x)-U_{c_{\lambda}}\left(x-c_{\lambda} t+x_{0}\right)\right| \rightarrow 0 \text { as } t \rightarrow+\infty,
$$

where $U_{c_{\lambda}}$ is the travelling front profile with speed $c_{\lambda}$, satisfying (1.5), and $x_{0}=-\lambda^{-1} \ln (\theta / M)$. This property implies that

$$
w_{*}\left(u_{0}\right)=w^{*}\left(u_{0}\right)=c_{\lambda}=\lambda+\frac{f^{\prime}(0)}{\lambda} .
$$

When $\lambda \geq \lambda^{*}$ in (1.8), McKean [16] and Kametaka [11] proved a similar convergence, namely

$$
\sup _{x \in \mathbb{R}}\left|u(t, x)-U_{c^{*}}\left(x-c^{*} t+m(t)\right)\right| \rightarrow 0 \text { as } t \rightarrow+\infty,
$$

where $m(t) / t \rightarrow 0$ as $t \rightarrow+\infty$. This implies (1.3) and leads to $w_{*}\left(u_{0}\right)=w^{*}\left(u_{0}\right)=c^{*}$. These above limits have been extended by Uchiyama [24] to general monostable functions $f$ fulfilling (1.2) and to front-like initial data satisfying $\lim _{x \rightarrow+\infty} u_{0}\left(x+x_{0}\right) / u_{0}(x)=e^{-\lambda x_{0}}$ for all $x_{0} \in \mathbb{R}$ (see also $[6,13,17,22,23]$ for further results and more precise convergence estimates). On the other hand, Bramson [4] and Lau [14] investigated spreading properties for more general front-like initial data, using probabilistic and PDE tools, when $f$ satisfies (1.2) and $f^{\prime}(s) \leq f^{\prime}(0)$. They proved that if $u_{0}$ is a front-like initial datum such that there exist $h>0$ and $0<\lambda<\lambda^{*}=\sqrt{f^{\prime}(0)}$ such that

$$
\lim _{x \rightarrow+\infty} \frac{1}{x} \ln \left(\int_{x}^{(1+h) x} u_{0}(y) d y\right)=-\lambda,
$$

then $w_{*}\left(u_{0}\right)=w^{*}\left(u_{0}\right)=c_{\lambda}=\lambda+f^{\prime}(0) / \lambda$. This result is more general than the one of Uchiyama [24], but it requires the nonlinearity $f$ to satisfy $f^{\prime}(s) \leq f^{\prime}(0)$. This property simplifies the analysis since it is known that the linearization near $u=0$ does govern the global dynamics of the equation in this case. Lastly, if $u_{0}$ is front-like and

$$
u_{0}(x) e^{\varepsilon x} \rightarrow+\infty \text { as } x \rightarrow+\infty \text { for all } \varepsilon>0,
$$

then it follows from the maximum principle and (1.10) that $w_{*}\left(u_{0}\right) \geq \varepsilon+f^{\prime}(0) / \varepsilon$ for all $\varepsilon>0$, whence $w_{*}\left(u_{0}\right)=w^{*}\left(u_{0}\right)=+\infty$. In this case, together with the Fisher-KPP assumption (1.4), Hamel and Roques [9] also computed the position of the level sets of the function $u(t, \cdot)$ as $t \rightarrow+\infty$, according to the precise asymptotic behaviour of $u_{0}$ at $+\infty$.

To sum up, the spreading speeds $w_{*}\left(u_{0}\right)$ and $w^{*}\left(u_{0}\right)$ are explicitly known when the frontlike initial data $u_{0}$ are exponentially decaying near $+\infty$, or when they fulfill (1.11) under the additional condition that $f$ satisfies $f^{\prime}(s) \leq f^{\prime}(0)$ for all $s \in[0,1]$. It is important to notice that, in all aforementioned examples, one has $w_{*}\left(u_{0}\right)=w^{*}\left(u_{0}\right)$. This leads to the following natural questions, that we investigate in the present paper: 
- Is it possible to compute $w_{*}\left(u_{0}\right)$ and $w^{*}\left(u_{0}\right)$ for more general initial conditions, given a nonlinearity $f$ satisfying (1.2) only?

- Is it always true that $w_{*}\left(u_{0}\right)=w^{*}\left(u_{0}\right)$ ?

Remark 1.3 Throughout the paper, the initial conditions $u_{0}$ are assumed to be front-like in the sense of Definition 1.1. Obviously, when $0 \leq u_{0} \leq 1, u_{0} \not \equiv 0$ and $u_{0}(x) \rightarrow 0$ as $x \rightarrow \pm \infty$, then left and right minimal and maximal spreading speeds could be defined and similar results as the ones stated in the next section could be obtained. One of the reasons lies on the fact that $u(t, x) \rightarrow 1$ as $t \rightarrow+\infty$ locally uniformly in $x \in \mathbb{R}$ (as a matter of fact, $\min _{|x| \leq c t} u(t, x) \rightarrow 1$ as $t \rightarrow+\infty$ for all $c \in\left[0, c^{*}\right)$, see [1]). Thus, the spreading properties to the left and to the right only depend on the behaviour of the tails of $u_{0}$ at $\pm \infty$. It would also have been natural to consider heterogeneous reaction-diffusion equations as well as equations in higher dimensions. We chose to present our results in the homogeneous one-dimensional setting for problem (1.1) for the sake of simplicity of the presentation, and also because this one-dimensional homogeneous framework already captures new and interesting complex propagation phenomena.

\section{Main results}

We first consider the class of front-like functions $u_{0}$ such that

$$
u_{0}(x)=O\left(e^{-\Lambda(x) x}\right) \text { as } x \rightarrow+\infty
$$

with $\lim _{x \rightarrow+\infty} \Lambda(x)=\lambda \in[0,+\infty]$. We first look for some conditions on $u_{0}$ which guarantee that $w_{*}\left(u_{0}\right)=w^{*}\left(u_{0}\right)=c_{\lambda}$. In other words, we want to know whether $u$ satisfies the same spreading property as the solution associated with the initial datum $x \mapsto \min \left(\sigma, \theta e^{-\lambda x}\right)$, for some $\sigma \in(0,1]$ and $\theta>0$. If $\lambda \in\left[\lambda^{*},+\infty\right]$, where $\lambda^{*}>0$ was defined in (1.7), then, as already emphasized, it follows from the maximum principle and [24] that $w_{*}\left(u_{0}\right)=w^{*}\left(u_{0}\right)=c^{*}$ (it is actually sufficient to suppose that $\left.\liminf _{x \rightarrow+\infty} \Lambda(x) \geq \lambda^{*}\right)$. We thus restrict ourselves to the case $0 \leq \lambda<\lambda^{*}$. The condition we will exhibit on $u_{0}$ depends on the function $x \mapsto \rho(x):=u_{0}(x) e^{\lambda x}$ (for $x$ sufficiently large). Basically, this condition requires the solution of the heat equation associated with the initial datum $\rho$ (extended by 1 in a neighborhood of $-\infty$ ) to be away from 0 along some rays in the $(t, x)$ variables. To make the arguments work, we shall use an additional assumption on the nonlinearity $f$ near 0 , which is fulfilled by $\mathcal{C}^{1+\gamma}$ functions. Namely, we assume that there exist $C>0, \gamma>0$ and $s_{0} \in(0,1)$ so that

$$
\forall s \in\left[0, s_{0}\right], f(s) \geq f^{\prime}(0) s-C s^{1+\gamma} .
$$

Theorem 2.1 Let $f$ satisfy (1.2) and (2.13), let $\lambda \in\left(0, \lambda^{*}\right)$ and let $u_{0}$ be a front-like function such that there exist $x_{0} \in \mathbb{R}$, a nonnegative bounded function $\rho:\left(x_{0},+\infty\right) \rightarrow[0,+\infty)$ and a function $\Lambda:\left(x_{0},+\infty\right) \rightarrow \mathbb{R}$ so that

$$
u_{0}(x)=\rho(x) e^{-\Lambda(x) x} \text { for a.e. } x>x_{0} \text { and } \Lambda(x) \rightarrow \lambda \text { as } x \rightarrow+\infty \text {. }
$$

Let $\bar{\rho}: \mathbb{R} \rightarrow[0,+\infty)$ be defined by $\bar{\rho}(x)=1$ for $x<x_{0}$ and $\bar{\rho}(x)=\rho(x)$ for $x>x_{0}$. Lastly, let $\zeta$ be the solution of the heat equation

$$
\left\{\begin{array}{l}
\partial_{t} \zeta-\partial_{x x} \zeta=0, \quad t>0, x \in \mathbb{R} \\
\zeta(0, x)=\bar{\rho}(x) \quad \text { for a.e. } x \in \mathbb{R} .
\end{array}\right.
$$


If there exists $\mu>0$ such that

$$
\liminf _{t \rightarrow+\infty} \zeta(t, c t)>0 \text { for all } c \in\left(c_{\lambda}-2 \lambda-\mu, c_{\lambda}-2 \lambda\right),{ }^{1}
$$

then

$$
w_{*}\left(u_{0}\right)=w^{*}\left(u_{0}\right)=c_{\lambda}=\lambda+\frac{f^{\prime}(0)}{\lambda} .
$$

Since $\inf _{t^{\prime} \geq t, x \in \mathbb{R}} \zeta\left(t^{\prime}, x\right) \geq \inf _{x \in \mathbb{R}} \zeta(t, x)$ for all $t>0$, from the maximum principle, an immediate consequence of Theorem 2.1 is the following

Corollary 2.2 With the same notations as in Theorem 2.1, the conclusion (2.16) holds if, instead of (2.15), the function $\zeta$ is such that there exists $T>0$ for which

$$
\inf _{x \in \mathbb{R}} \zeta(T, x)>0 .
$$

Remark 2.3 In this remark, we discuss the relationship between the assumptions (2.15) and (2.17) and we give some equivalent formulations. First, condition (2.17) clearly implies (2.15), but these two properties are not equivalent. Namely, one can construct classes of explicit examples of functions $\rho$ for which the condition (2.15) is fulfilled and (2.17) is not. As an archetype, choose $x_{0}=0$ and

$$
\rho(x)=0 \text { for } x \in \bigcup_{n \in \mathbb{N}}\left(2 n^{5}-n, 2 n^{5}+n\right)
$$

and $\rho(x)=1$ otherwise. One has $0<\zeta(t, x)<1$ for all $t>0$ and $x \in \mathbb{R}$, and $\zeta\left(t, 2 n^{5}\right) \rightarrow 0$ as $n \rightarrow+\infty$ for all $t>0$. Hence,

$$
\inf _{x \in \mathbb{R}} \zeta(t, x)=0
$$

for all $t>0$ and condition (2.17) is not fulfilled. On the other hand, for any fixed $c \in \mathbb{R}$, there holds

$$
0<1-\zeta(t, c t) \leq \frac{1}{\sqrt{\pi}} \int_{-\infty}^{+\infty} \mathbf{1}_{\cup_{n \in \mathbb{N}} I_{n, t}}(z) e^{-z^{2}} d z \text { for all } t>0,
$$

where $I_{n, t}$ is the interval

$$
I_{n, t}=\left(\frac{2 n^{5}-n-c t}{\sqrt{4 t}}, \frac{2 n^{5}+n-c t}{\sqrt{4 t}}\right) .
$$

Fix $\varepsilon>0$ and let $A>0$ be such that

$$
\pi^{-1 / 2} \int_{\mathbb{R} \backslash(-A, A)} e^{-z^{2}} d z \leq \varepsilon
$$

If $I_{n, t}$ intersects $(-A, A)$, then $\left(2 n^{5}-n-c t\right) / \sqrt{4 t}<A$, whence $n^{5} \leq(|c|+1) t$ for large $t$. Since the length of $I_{n, t}$ is $n / \sqrt{t}$, there exists $t_{0}>0$ such that, for all

\footnotetext{
${ }^{1}$ Notice that $c_{\lambda}-2 \lambda=\sqrt{c_{\lambda}^{2}-4 f^{\prime}(0)}>0$.
} 
$t \geq t_{0}$, the sum of the lengths of the intervals $I_{n, t}$ intersecting $(-A, A)$ is at most $(|c|+1)^{1 / 5} t^{1 / 5} \times(|c|+1)^{1 / 5} t^{1 / 5} / \sqrt{t}=(|c|+1)^{2 / 5} t^{-1 / 10}$. Thus, for all $t \geq t_{0}$,

$$
\frac{1}{\sqrt{\pi}} \int_{-A}^{A} \mathbf{1}_{\cup_{n \in \mathbb{N}} I_{n, t}}(z) e^{-z^{2}} d z \leq \frac{(|c|+1)^{2 / 5} t^{-1 / 10}}{\sqrt{\pi}},
$$

whence $\lim \sup _{t \rightarrow+\infty} 1-\zeta(t, c t) \leq \varepsilon$. Finally,

$$
\lim _{t \rightarrow+\infty} \zeta(t, c t)=1
$$

for every $c \in \mathbb{R}$ and (2.15) holds. Let us now give some equivalent formulations to (2.15) and (2.17). Notice that, by definition of $\bar{\rho}$, there holds $\zeta(t, x) \rightarrow 1$ as $x \rightarrow-\infty$ for every $t>0$. Since $\zeta$ is continuous and positive on $(0,+\infty) \times \mathbb{R}$, it follows that condition (2.15) is equivalent to

$$
\liminf _{t \rightarrow+\infty}\left(\inf _{x \leq c t} \zeta(t, x)\right)>0 \text { for all } c<c_{\lambda}-2 \lambda .
$$

Indeed, for every $c \in\left(c_{\lambda}-2 \lambda-\mu, c_{\lambda}-2 \lambda\right)$, there are $T>0$ and $\eta>0$ such that $\zeta(t, c t) \geq \eta$ for all $t \geq T$; even if it means decreasing $\eta>0$, one can assume that $\zeta(T, x) \geq \eta$ for all $x \leq c T$; thus the maximum principle yields $\zeta(t, x) \geq \eta$ for all $t \geq T$ and $x \leq c t$. Actually, the same arguments imply that $(2.15)$ is fulfilled if

$$
\liminf _{t \rightarrow+\infty} u\left(t,\left(c_{\lambda}-2 \lambda\right) t\right)>0 .
$$

Notice also that $(2.17)$ is equivalent to $\inf _{x \in \mathbb{R}} \zeta(t, x)>0$ for all $t>0$ : indeed, if there exist $t_{0}>0$ and a sequence $\left(x_{n}\right)_{n \in \mathbb{N}}$ of real numbers such that $\zeta\left(t_{0}, x_{n}\right) \rightarrow 0$ as $n \rightarrow+\infty$, then the Schauder parabolic estimates and the strong parabolic maximum principle imply that, up to extraction of a subsequence, $\zeta\left(t, x+x_{n}\right) \rightarrow 0$ as $n \rightarrow+\infty$ locally uniformly in $(t, x) \in(0,+\infty) \times \mathbb{R}$. Lastly, observe that, by linearity of the heat equation and by the maximum principle, conditions (2.15) and (2.17) remain unchanged if the function $\bar{\rho}$ is set to be equal to any given positive real number $\eta>0$, instead of 1 , on $\left(-\infty, x_{0}\right)$.

Under only the monostability and behaviour-at-0 properties (1.2) and (2.13), Theorem 2.1 and Corollary 2.2 give sufficient conditions on $u_{0}$ for the solution $u$ of (1.1) to spread at speed $c_{\lambda}$. Let us now discuss the role of the various assumptions. First, without the boundedness of $\rho$, the conclusion (2.16) may not hold: for instance, if $\rho(x)=e^{\varepsilon x}$ for $x>x_{0}$ with $\varepsilon \in(0, \lambda)$, then, by writing $u_{0}(x)=e^{-\widetilde{\Lambda}(x) x}$ for a.e. $x>x_{0}$ with $\widetilde{\Lambda}(x)=\Lambda(x)-\varepsilon$, Corollary 2.2 yields $w_{*}\left(u_{0}\right)=w^{*}\left(u_{0}\right)=c_{\lambda-\varepsilon}>c_{\lambda}$. Similarly, without (2.15) or (2.17), (2.16) may not hold: for instance, if $\rho(x)=e^{-\varepsilon x}$ for $x>x_{0}$ with $\varepsilon \in\left(0, \lambda^{*}-\lambda\right)$, then $w_{*}\left(u_{0}\right)=w^{*}\left(u_{0}\right)=c_{\lambda+\varepsilon}<c_{\lambda}$, whereas conditions (2.15) and (2.17) are not fulfilled since, for every $c>0$,

$$
0<\zeta(t, c t)=\frac{1}{\sqrt{4 \pi t}} \int_{-\infty}^{+\infty} e^{-\frac{(y-c t)^{2}}{4 t}} \bar{\rho}(y) d y=\frac{1}{\sqrt{\pi}} \int_{-\infty}^{+\infty} e^{-z^{2}} \bar{\rho}(c t+z \sqrt{4 t}) d z \rightarrow 0 \text { as } t \rightarrow+\infty
$$

from Lebesgue's dominated convergence theorem. However, the conditions (2.15) or (2.17) are not necessary in general. That is, (2.16) may hold without (2.15) or (2.17): for instance, if $\rho$ is any positive measurable function on $\left(x_{0},+\infty\right)$ such that

$$
\rho(x) \rightarrow 0 \text { and }|\ln \rho(x)|=o(x) \text { as } x \rightarrow+\infty,
$$


then as above $\zeta(t, c t) \rightarrow 0$ as $t \rightarrow+\infty$ for all $c>0$, whence (2.15) and (2.17) are not fulfilled; nevertheless conclusion (2.16) still holds since Corollary 2.2 can be applied by writting $u_{0}$ as $u_{0}(x)=e^{-\widetilde{\Lambda}(x) x}$ for a.e. $x \geq x_{0}$ with

$$
\widetilde{\Lambda}(x)=\Lambda(x)-\frac{\ln \rho(x)}{x} \rightarrow \lambda \text { as } x \rightarrow+\infty .
$$

A corollary of Theorem 2.1 concerns the case of functions $\rho$ being asymptotically positive in average at $+\infty$, by which we mean

$$
\liminf _{x \rightarrow+\infty}\left(\inf _{h \geq \beta \sqrt{x}} h^{-1} \int_{x}^{x+h} \rho(z) d z\right)>0 \text { for some } \beta>0 .
$$

Corollary 2.4 Let $f, \lambda, u_{0}, x_{0}, \rho$ and $\Lambda$ be as in Theorem 2.1 and assume that $\rho$ is asymptotically positive in average at $+\infty$. Then (2.16) holds.

Let us now give some further applications of the above results. As a particular case, Corollary 2.4 covers the case of functions $\rho$ which can extended to a uniformly continuous nonnegative periodic, almost-periodic or uniquely ergodic function $\rho \in L^{\infty}(\mathbb{R})$ having a positive average. $^{2}$ Under the assumptions of Corollary 2.4, it is easy to check that $u_{0}$ satisfies the condition (1.11) of Bramson [4] and Lau [14]. Hence, if, in addition to (1.2) and (2.13), the function $f$ is such that $f^{\prime}(s) \leq f^{\prime}(0)$, Corollary 2.4 gives then an alternate approach of that of Bramson and Lau. Another enlightening application of the above results is the following one. Let $0<\lambda_{1}<\lambda_{2}<\lambda^{*}$ be fixed, let $\rho_{1}$ and $\rho_{2}$ be two given bounded nonnegative periodic functions with positive averages, let $u_{1,0}$ and $u_{2,0}$ be two given front-like functions such that

$$
u_{1,0}(x)=\rho_{1}(x) e^{-\lambda_{1} x}
$$

and

$$
u_{2,0}(x)=\rho_{2}(x) e^{-\lambda_{2} x}
$$

for large $x$, and let $u_{1}$ and $u_{2}$ be the solutions of (1.1) with initial conditions $u_{1,0}$ and $u_{2,0}$, respectively. It follows from Corollary 2.4 that $u_{1}$ and $u_{2}$ spread at the speeds $c_{\lambda_{1}}=\lambda_{1}+f^{\prime}(0) / \lambda_{1}$ and $c_{\lambda_{2}}=\lambda_{2}+f^{\prime}(0) / \lambda_{2}$, respectively. Let now $u_{0}$ be a front-like function such that

$$
u_{0}(x)=\rho_{1}(x) e^{-\lambda_{1} x}+\rho_{2}(x) e^{-\lambda_{2} x} \text { for large } x
$$

and let $u$ be the solution of (1.1) with initial condition $u_{0}$. Since $u_{0}$ is equal to a linear combination of the functions $u_{1,0}$ and $u_{2,0}$ near $+\infty$, one could have thought that $u$ would have spread at a speed which would have been a sort of average of $c_{\lambda_{1}}$ and $c_{\lambda_{2}}$. This is actually not the case, since Theorem 2.1 implies that $w_{*}\left(u_{0}\right)=w^{*}\left(u_{0}\right)=c_{\lambda_{1}}$. In other words, $u$ spreads at the largest speed, that is the one given only from the slowest exponential decay. Indeed, for large $x, u_{0}(x)=\rho(x) e^{-\lambda_{1} x}$, where $\rho(x)=\rho_{1}(x)+\rho_{2}(x) e^{-\left(\lambda_{2}-\lambda_{1}\right) x}$ is bounded near $+\infty$;

\footnotetext{
${ }^{2} \mathrm{~A}$ uniformly continuous bounded function $\rho$ is uniquely ergodic if the limit $\lim _{h \rightarrow+\infty} h^{-1} \int_{x}^{x+h} F\left(\tau_{z} \rho\right) d z$ exists uniformly with respect to $x \in \mathbb{R}$, where $\tau_{z} \rho=\rho(\cdot+z)$ and $F$ is any continuous real-valued map defined on the closure of the set of all functions $\tau_{z} \rho$ in the sense of local uniform convergence, see [3, 18]. The periodic, almost-periodic or uniquely ergodic functions $\rho$ have a (uniform) average $\rho_{m} \in \mathbb{R}$ in the sense that $h^{-1} \int_{x}^{x+h} \rho(z) d z \rightarrow \rho_{m}$ as $h \rightarrow+\infty$ uniformly in $x \in \mathbb{R}$.
} 
since $\rho \geq \rho_{1} \geq 0$ and $\rho_{1}$ has a positive average, the condition (2.17) is fulfilled and the conclusion (2.16) holds with $\lambda=\lambda_{1}$.

We point out that, in Theorem 2.1 or Corollaries 2.2 or 2.4 , the function $\rho$ may vanish on sequences of sets with positive measure on $[A,+\infty)$ for all large $A$, in which case the function $u_{0}$ cannot be bounded from below by a positive constant times any function $e^{-\lambda x}$ for large $x$. A typical example is when $\rho$ is periodic and vanishes periodically. However, for the conclusion (2.16) to hold, the function $\rho$ cannot be too close to 0 on a too large set, this is roughly speaking the meaning of the sort of homogenization conditions (2.15) and (2.17). The simplest example is when $\rho$ is periodic: $\rho$ may vanish periodically but, unless it vanishes almost everywhere, the spreading speeds $w_{*}\left(u_{0}\right)$ and $w^{*}\left(u_{0}\right)$ are equal to $c_{\lambda}$.

When, in Corollary 2.2, the function $\Lambda$ is equal to the constant $\lambda$, the method we use gives actually more than (2.16), under the assumption (2.17):

Proposition 2.5 Let $f, \lambda, u_{0}, x_{0}, \rho$ and $\Lambda$ be as in Corollary 2.2 and assume that $\Lambda=\lambda$ on $\left[x_{0},+\infty\right)$ and that $(2.17)$ holds. Then there are $x_{1}, x_{2} \in \mathbb{R}$ such that, uniformly in $x \in \mathbb{R}$,

$$
U_{c_{\lambda}}\left(x+x_{1}\right) \leq \liminf _{t \rightarrow+\infty} u\left(t, x+c_{\lambda} t\right) \leq \limsup _{t \rightarrow+\infty} u\left(t, x+c_{\lambda} t\right) \leq U_{c_{\lambda}}\left(x+x_{2}\right) .
$$

This result means that the solution $u$ is asymptotically almost trapped between two shifts of a travelling front moving with speed $c_{\lambda}=\lambda+f^{\prime}(0) / \lambda$. In the case when $\Lambda$ depends on $x$, the conclusion is not true in general, see the comment after Corollary 2.6 on the position of the level sets of $u$ at large time. However, even when $\Lambda$ is constant, formula (2.18) does not mean that $u\left(t, \cdot+c_{\lambda} t\right)$ is truly trapped between two shifts of $U_{c_{\lambda}}$, even for large $t$. Indeed, for instance, if $0<\operatorname{esssup}_{\mathbb{R}} u_{0}=M_{0}<1$, then $\sup _{\mathbb{R}} u(t, \cdot) \leq M(t)$ for all $t \geq 0$, where $\dot{M}(t)=f(M(t))$ for all $t \geq 0$ and $M(0)=M_{0}$. Since $M(t)<1$ for all $t \geq 0$ and since $U_{c_{\lambda}}(-\infty)=1$, the function $u\left(t, \cdot+c_{\lambda} t\right)$ can never be larger than any shift of $U_{c_{\lambda}}$. Proposition 2.5 does not mean either that $u\left(t, \cdot+c_{\lambda} t\right)$ converges to a shift of the front $U_{c_{\lambda}}$. The solution may well oscillate without converging between two shifts of the front $U_{c_{\lambda}}$, as proved in $[2,15]$ when $f$ is concave.

Lastly, when $u_{0}$ is not exponentially bounded as $x \rightarrow+\infty$, in the sense of (1.12), then $w_{*}\left(u_{0}\right)=w^{*}\left(u_{0}\right)=+\infty$, as already noticed. Theorem 2.1 and the condition $(2.15)$ can be adapted to the case $\lambda=0$, as the following corollary shows.

Corollary 2.6 If, in Theorem 2.1, one sets $\lambda=0$ and (2.15) is replaced with the existence of a real number $\gamma$ such that $\liminf _{t \rightarrow+\infty} u(t, c t)>0$ for all $c>\gamma$, then $w_{*}\left(u_{0}\right)=w^{*}\left(u_{0}\right)=+\infty$.

It is possible to reformulate the above results in terms of the level sets of the solution $u$ of the Cauchy problem (1.1). Namely, given a front-like initial condition $u_{0}$, define the level set of $u$ for a value $m \in(0,1)$ at a time $t>0$, as follows:

$$
E_{m}(t)=\{x \in \mathbb{R}, u(t, x)=m\} .
$$

For a given $m \in(0,1)$, this set can be empty, but it is non-empty and compact when $t$ is sufficiently large. From Definition 1.2 and under the hypotheses of Theorem 2.1 with $0<\lambda<\lambda^{*}$ (resp. Corollary 2.6 with $\lambda=0$ ), we can reformulate the conclusions into:

$$
\forall m \in(0,1), \lim _{t \rightarrow+\infty} \frac{1}{t} \min E_{m}(t)=\lim _{t \rightarrow+\infty} \frac{1}{t} \max E_{m}(t)=c_{\lambda}=\lambda+\frac{f^{\prime}(0)}{\lambda}
$$


with the convention that $c_{0}=+\infty$. In other words, for any $m \in(0,1)$ and any family of real numbers $\left(x_{m}(t)\right)_{t>0}$ such that $u\left(t, x_{m}(t)\right)=m$ for large $t$, then $x_{m}(t) / t \rightarrow c_{\lambda}$ as $t \rightarrow+\infty$. Thus, the quantity $c_{\lambda}$ is the asymptotic time-averaged speed of all level sets of $u$. As far as Proposition 2.5 is concerned, its conclusion (2.18) implies in particular that, for all $m \in(0,1)$,

$$
\limsup _{t \rightarrow+\infty}\left|\max E_{m}(t)-c_{\lambda} t\right|<+\infty \text { and } \limsup _{t \rightarrow+\infty}\left|\min E_{m}(t)-c_{\lambda} t\right|<+\infty
$$

Property (2.20) is clearly stronger than (2.19). Both (2.19) and (2.20) also yield formula (2.16), since, as it can be easily seen, $\liminf _{x \rightarrow-\infty} u(t, x) \rightarrow 1$ as $t \rightarrow+\infty$ and $\lim _{x \rightarrow+\infty} u(t, x)=0$ for all $t \geq 0$. However, it is worth noticing here that, in general, the assumptions of Theorem 2.1 do not guarantee that the level sets $E_{m}(t)$ stay at finite distance as $t \rightarrow+\infty$ from the position $c_{\lambda} t$ for each fixed $m \in(0,1)$. For instance, if $u_{0}$ is front-like and $u_{0}(x)=e^{-\Lambda(x) x}$ for large $x$ with $\lim _{x \rightarrow+\infty} \Lambda(x)=\lambda \in\left(0, \lambda^{*}\right)$ and $\lim _{x \rightarrow+\infty}(\Lambda(x)-\lambda) x=+\infty($ resp. $-\infty)$, then $u_{0}(x) \leq \eta e^{-\lambda x}$ (resp. $u_{0}(x) \geq \eta e^{-\lambda x}$ ) for $x$ large enough, for every fixed $\eta>0$; thus, it follows from the comparison principle and the general convergence results (1.9), that

$$
\limsup _{t \rightarrow+\infty}\left(\sup _{x \in \mathbb{R}}\left(u(t, x)-U_{c_{\lambda}}\left(x-c_{\lambda} t+A\right)\right)\right) \leq 0
$$

(resp.

$$
\left.\liminf _{t \rightarrow+\infty}\left(\inf _{x \in \mathbb{R}}\left(u(t, x)-U_{c_{\lambda}}\left(x-c_{\lambda} t+A\right)\right)\right) \geq 0\right)
$$

for every $A \in \mathbb{R}$, whence

$$
\max E_{m}(t)-c_{\lambda} t \rightarrow-\infty
$$

(resp.

$$
\left.\min E_{m}(t)-c_{\lambda} t \rightarrow+\infty\right)
$$

as $t \rightarrow+\infty$ for all value $m \in(0,1)$, while $w_{*}\left(u_{0}\right)=w^{*}\left(u_{0}\right)=c_{\lambda}$ from Theorem 2.1. On the other hand, if $|\Lambda(x)-\lambda|=O\left(x^{-1}\right)$ as $x \rightarrow+\infty$ and if (2.17) is fulfilled, then Proposition 2.5 and the maximum principle imply that (2.18) holds, whence (2.20).

In all above results, the solutions $u$ of (1.1) have a well defined spreading speed, that is $w_{*}\left(u_{0}\right)=w^{*}\left(u_{0}\right)$, and this quantity is explicitely expressed in terms of the asymptotic behaviour of the front-like initial condition at $+\infty$. We now exhibit a class of front-like initial data $u_{0}$ for which $w_{*}\left(u_{0}\right)<w^{*}\left(u_{0}\right)$, and the difference $w^{*}\left(u_{0}\right)-w_{*}\left(u_{0}\right)$ may actually be arbitrarily large. We not only prove that for some range of speeds $c$, the functions $t \mapsto u(t, c t+x)$ do not converge to anything as $t \rightarrow+\infty$, but also that their $\omega$-limit sets are the whole interval $[0,1]$. We recall that the $\omega$-limit set as $t \rightarrow+\infty$ of a function $t \mapsto g(t) \in[0,1]$ defined in a neighborhood of $+\infty$ is the set of all $s \in[0,1]$ for which there exists a sequence $t_{n} \rightarrow+\infty$ such that $g\left(t_{n}\right) \rightarrow s$ as $n \rightarrow+\infty$. Given a function $f$ satisfying (1.2), we denote

$$
M_{f}=\max _{s \in[0,1]} f^{\prime}(s)>0 .
$$

From comparisons with KPP-type nonlinearities, it follows that $c^{*} \leq 2 \sqrt{M_{f}}$, where $c^{*}$ is the minimal speed of travelling fronts with nonlinearity $f$ (see also [8]). 
Theorem 2.7 Let $f$ satisfy (1.2) and (2.13) and let $\gamma_{1}<\gamma_{2}$ be given in the interval $\left[2 \sqrt{M_{f}},+\infty\right]$. Then there exists a front-like function $u_{0}$ such that

$$
\gamma_{1}=w_{*}\left(u_{0}\right)<w^{*}\left(u_{0}\right)=\gamma_{2} .
$$

Furthermore, for any $c \in\left(\gamma_{1}, \gamma_{2}\right)$, any $x \in \mathbb{R}$ and any $m \in(0,1)$, the $\omega$-limit set of the function $t \mapsto u(t, c t+x)$ as $t \rightarrow+\infty$ is equal to the whole interval $[0,1]$ and the $\omega$-limit sets of the functions $t \mapsto t^{-1} \min E_{m}(t)$ and $t \mapsto t^{-1} \max E_{m}(t)$ are equal to the whole interval $\left[\gamma_{1}, \gamma_{2}\right]$.

The initial data $u_{0}$ are constructed in such a way that they oscillate as $x \rightarrow+\infty$ between the two exponential functions $e^{-\lambda_{1} x}$ and $e^{-\lambda_{2} x}$ on larger and larger space-intervals, with $\gamma_{1}=c_{\lambda_{1}}$ (or $\lambda_{1}=\lambda^{*}$ if $\gamma_{1}=c^{*}$ ) and $\gamma_{2}=c_{\lambda_{2}}$. The proof then shows that the solution $u$ of (1.1) oscillates on larger and larger time-intervals between two approximate solutions moving with speeds close to $\gamma_{1}$ and $\gamma_{2}$, so that the averaged speeds of the level sets, namely min $E_{m}(t) / t$ and $\max E_{m}(t) / t$, oscillate infinitely many times between $\gamma_{1}$ and $\gamma_{2}$. Therefore, the level sets do not converge in speed to any real number as $t \rightarrow+\infty$. It is worth noticing that, for such monostable problems, this completely new and highly non-trivial oscillating dynamics is present even in the simplest case of the one-dimensional homogeneous equation (1.1). It holds for general monostable functions $f$ satisfying (1.2) and (2.13), provided that the chosen speeds $\gamma_{1}$ and $\gamma_{2}$ are large enough. Notice that, in the case when $f^{\prime}(s) \leq f^{\prime}(0)$, then $M_{f}=f^{\prime}(0)$ and $c^{*}=2 \sqrt{M_{f}}$, whence the speeds $\gamma_{1}$ and $\gamma_{2}$ can take any values between $c^{*}$ and $+\infty$.

The proofs of the above results rely firstly on the maximum principle and on the construction of suitable sub- and supersolutions for the (1.1). The gaussian decay of the heat kernel plays a crucial role in Theorem 2.7. We have to estimate sharply the time-depending behaviour of $u(t, x)$ as $x \rightarrow+\infty$ and we prove that these tails force the solution to spread at the desired approximated speeds on large time-intervals. We point out that, even if the spreading properties are determined through the asymptotic behaviour of $u(t, x)$ as $x \rightarrow+\infty$, that is as $u \rightarrow 0$, the function $f$ does not need to be concave or of the KPP type (1.4).

\section{The case when the spreading speed is unique}

This section is devoted to the proof of Theorem 2.1 and its corollaries. It is based on the construction of sub- and supersolutions moving asymptotically at the speed $c_{\lambda}$, and on the basic interpretation of the solutions of the linearized problem (3.21) below in terms of the solutions of the heat equation (2.14).

Proof of Theorem 2.1. Let $\varepsilon \in(0, \lambda)$ be arbitrary. As $0<\lambda-\varepsilon<\lambda<\lambda^{*}$, one has $(\lambda-\varepsilon)^{2}-(\lambda-\varepsilon) c^{*}+f^{\prime}(0)>0$. In other words, $c_{\lambda-\varepsilon}>c^{*}$. As recalled in the introduction, there exists a travelling front solution $U_{c_{\lambda-\varepsilon}}\left(x-c_{\lambda-\varepsilon} t\right)$ of (1.1) with speed $c_{\lambda-\varepsilon}$, and such that $U_{c_{\lambda-\varepsilon}}(x) \sim M e^{-(\lambda-\varepsilon) x}$ as $x \rightarrow+\infty$, for some $M>0$. Since $\rho$ is bounded and $\Lambda(x) \rightarrow \lambda$ as $x \rightarrow+\infty$, there exists $\widetilde{M}>0$ such that $u_{0}(x) \leq \bar{u}_{0}(x)$ for a.e. $x \in \mathbb{R}$, where

$$
\bar{u}_{0}(x)=\min \left(1, \widetilde{M} e^{-(\lambda-\varepsilon) x}\right) .
$$

Let now $\bar{u}$ be the solution of (1.1) with initial condition $\bar{u}_{0}$. Because of (1.9), there exists a real number $x_{2}$ such that

$$
\sup _{x \in \mathbb{R}}\left|\bar{u}(t, x)-U_{c_{\lambda-\varepsilon}}\left(x-c_{\lambda-\varepsilon} t+x_{2}\right)\right| \rightarrow 0 \text { as } t \rightarrow+\infty .
$$


But the maximum principle yields $u(t, x) \leq \bar{u}(t, x)$ for all $t>0$ and $x \in \mathbb{R}$. Thus, for any $c>c_{\lambda-\varepsilon}$,

$$
\begin{aligned}
0 \leq \limsup _{t \rightarrow+\infty}\left(\max _{x \geq c t} u(t, x)\right) & \leq \limsup _{t \rightarrow+\infty}\left(\max _{x \geq c t} \bar{u}(t, x)\right) \\
& =\limsup _{t \rightarrow+\infty}\left(\max _{x \geq c t} U_{c_{\lambda-\varepsilon}}\left(x-c_{\lambda-\varepsilon} t+x_{2}\right)\right) \\
& =\limsup _{t \rightarrow+\infty} U_{c_{\lambda-\varepsilon}}\left(c t-c_{\lambda-\varepsilon} t+x_{2}\right)=0
\end{aligned}
$$

whence $w^{*}\left(u_{0}\right) \leq c_{\lambda-\varepsilon}$. By passing to the limit as $\varepsilon \rightarrow 0^{+}$, one gets that $w^{*}\left(u_{0}\right) \leq c_{\lambda}$.

In order to prove the left inequality in (2.16), fix an arbitrary $\varepsilon>0$ such that $\lambda+\varepsilon<\lambda^{*}$ and consider the solution $\xi$ of the linear problem

$$
\begin{cases}\partial_{t} \xi-\partial_{x x} \xi=f^{\prime}(0) \xi, & t>0, x \in \mathbb{R} \\ \xi(0, x)=\bar{\rho}(x) e^{-(\lambda+\varepsilon) x} & \text { for a.e. } x \in \mathbb{R} .\end{cases}
$$

From the definition of $c_{\lambda+\varepsilon}$, the maximum principle yields

$$
\xi(t, x) \leq\|\bar{\rho}\|_{L^{\infty}(\mathbb{R})} e^{-(\lambda+\varepsilon)\left(x-c_{\lambda+\varepsilon} t\right)} \text { for all } t>0 \text { and } x \in \mathbb{R} .
$$

The first key-point here is to observe that the function

$$
(t, x) \mapsto e^{-(\lambda+\varepsilon)\left(x-c_{\lambda+\varepsilon} t\right)} \zeta(t, x-2(\lambda+\varepsilon) t)
$$

solves (3.21), since $\zeta$ solves (2.14). Thus, by uniqueness, one has

$$
\xi(t, x)=e^{-(\lambda+\varepsilon)\left(x-c_{\lambda+\varepsilon} t\right)} \zeta(t, x-2(\lambda+\varepsilon) t) \text { for all } t>0 \text { and } x \in \mathbb{R} .
$$

Set now $P(\beta)=\beta^{2}-\beta c_{\lambda+\varepsilon}+f^{\prime}(0)$ for all $\beta \in \mathbb{R}$. This function $P$ is decreasing on the interval $[0, \lambda+\varepsilon]$ since $\lambda+\varepsilon>0$ is its smallest simple zero, and one has $2(\lambda+\varepsilon)<c_{\lambda+\varepsilon}$. Remember that $s_{0} \in(0,1), \gamma>0$ and $C>0$ are given in (2.13). Choose $\theta>0$ and $\kappa \in(0,1]$ small enough so that

$$
(1+\gamma)(\lambda+\varepsilon) \geq \lambda+\varepsilon+\theta
$$

and

$$
\left(c_{\lambda+\varepsilon}-2(\lambda+\varepsilon)-\theta\right) \theta \geq \kappa^{\gamma} .
$$

Next, let $x_{1} \geq \max \left(x_{0}, 0\right)$ be chosen so that $\Lambda(x) \leq \lambda+\varepsilon$ for a.e. $x \geq x_{1}$. Owing to (3.22), choose now $A>0$ large enough so that $A \geq C\|\bar{\rho}\|_{L^{\infty}(\mathbb{R})}^{1+\gamma}$,

$$
\forall(t, x) \in(0,+\infty) \times \mathbb{R},\left(\xi(t, x)>A e^{-(\lambda+\varepsilon+\theta)\left(x-c_{\lambda+\varepsilon} t\right)}\right) \Longrightarrow\left(x \geq \max \left(c_{\lambda+\varepsilon} t, x_{1}\right)\right),
$$

and

$$
\kappa\left(\xi(t, x)-A e^{-(\lambda+\varepsilon+\theta)\left(x-c_{\lambda+\varepsilon} t\right)}\right) \leq s_{0} \text { for all } t>0 \text { and } x \in \mathbb{R}
$$

Lastly, set

$$
\underline{u}(t, x)=\max \left(0, \kappa\left(\xi(t, x)-A e^{-(\lambda+\varepsilon+\theta)\left(x-c_{\lambda+\varepsilon} t\right)}\right)\right)
$$


in $[0,+\infty) \times \mathbb{R}$. It follows that

$$
\sup _{(t, x) \in(0,+\infty) \times \mathbb{R}} \underline{u}(t, x) \leq s_{0}
$$

and

$$
\begin{aligned}
\Omega & =\{(t, x) \in(0,+\infty) \times \mathbb{R}, \underline{u}(t, x)>0\} \\
& \subset\left\{(t, x) \in(0,+\infty) \times \mathbb{R}, x \geq \max \left(c_{\lambda+\varepsilon} t, x_{1}\right)\right\} .
\end{aligned}
$$

Let us then check that $\underline{u}$ is a subsolution for problem (1.1). When $(t, x) \in \Omega$, one has

$$
\begin{aligned}
& \partial_{t} \underline{u}(t, x)-\partial_{x x} \underline{u}(t, x)-f^{\prime}(0) \underline{u}(t, x) \\
&=\left((\lambda+\varepsilon+\theta)^{2}-(\lambda+\varepsilon+\theta) c_{\lambda+\varepsilon}+f^{\prime}(0)\right) \kappa A e^{-(\lambda+\varepsilon+\theta)\left(x-c_{\lambda+\varepsilon} t\right)} \\
&=-\left(c_{\lambda+\varepsilon}-2(\lambda+\varepsilon)-\theta\right) \theta \kappa A e^{-(\lambda+\varepsilon+\theta)\left(x-c_{\lambda+\varepsilon} t\right)} \\
& \leq-\left(c_{\lambda+\varepsilon}-2(\lambda+\varepsilon)-\theta\right) \theta \kappa A e^{-(1+\gamma)(\lambda+\varepsilon)\left(x-c_{\lambda+\varepsilon} t\right)} \\
& \leq-\kappa^{1+\gamma} A e^{-(1+\gamma)(\lambda+\varepsilon)\left(x-c_{\lambda+\varepsilon} t\right)} \\
& \leq-C \underline{u}(t, x)^{1+\gamma}
\end{aligned}
$$

from (3.22) and the choice of $\varepsilon, \kappa$ and $A$. Therefore,

$$
\partial_{t} \underline{u}-\partial_{x x} \underline{u} \leq f(\underline{u}) \text { in } \Omega
$$

because of (2.13). It also follows from the definition of $\xi(0, \cdot)$ and from the choices of $x_{1}$ and $A$ and the inequality $0<\kappa \leq 1$, that $\underline{u}(0, x) \leq u(0, x)$ for a.e. $x \in \mathbb{R}$. Summing up, as $\underline{u}=0$ in $(0,+\infty) \times \mathbb{R} \backslash \Omega$, the function $\underline{u}$ is a subsolution of (1.1). Thus

$$
u(t, x) \geq \underline{u}(t, x) \text { for all }(t, x) \in(0,+\infty) \times \mathbb{R}
$$

from the maximum principle.

Observe now that $c_{\lambda+\varepsilon}-2(\lambda+\varepsilon)<c_{\lambda}-2 \lambda$ and pick any $c$ such that

$$
c_{\lambda+\varepsilon}-2(\lambda+\varepsilon)<c<c_{\lambda}-2 \lambda .
$$

From assumption (2.15) and Remark 2.3, there holds

$$
\nu:=\liminf _{t \rightarrow+\infty}\left(\inf _{x \leq c t} \zeta(t, x)\right)>0 .
$$

Take any $B \in \mathbb{R}$ such that $A e^{-\theta B}<\nu / 2$. It follows then from (3.23), (3.24) and (3.25) that

$$
\begin{aligned}
\liminf _{t \rightarrow+\infty} u & \left(t, c_{\lambda+\varepsilon} t+B\right) \\
& \geq \liminf _{t \rightarrow+\infty} \underline{u}\left(t, c_{\lambda+\varepsilon} t+B\right) \\
& \geq \kappa \liminf _{t \rightarrow+\infty} \xi\left(t, c_{\lambda+\varepsilon} t+B\right)-\kappa A e^{-(\lambda+\varepsilon+\theta) B} \\
& =\kappa e^{-(\lambda+\varepsilon) B}\left(\liminf _{t \rightarrow+\infty} \zeta\left(t, c_{\lambda+\varepsilon} t-2(\lambda+\varepsilon) t+B\right)-A e^{-\theta B}\right) \\
& \geq \kappa e^{-(\lambda+\varepsilon) B}\left(\nu-A e^{-\theta B}\right) \\
& >\omega:=\frac{\kappa \nu e^{-(\lambda+\varepsilon) B}}{2}>0 .
\end{aligned}
$$


On the other hand, the maximum principle implies that $\liminf _{x \rightarrow-\infty} u(t, x) \geq \theta(t)$ for all $t>0$, where $\dot{\theta}(t)=f(\theta(t))$ in $[0,+\infty)$ and $\theta(0)=\liminf _{x \rightarrow-\infty}\left(\operatorname{essinf}_{(-\infty, x)} u_{0}\right)>0$. Hence,

$$
\liminf _{x \rightarrow-\infty} u(t, x) \geq \theta(t)>0 \text { for all } t>0 .
$$

Let $T>0$ be such that

$$
u\left(t, c_{\lambda+\varepsilon} t+B\right) \geq \omega \text { for all } t \geq T .
$$

From the previous arguments and the positivity and continuity of $u$ on $(0,+\infty) \times \mathbb{R}$, there holds

$$
\inf _{x \leq c_{\lambda+\varepsilon} T+B} u(T, x)>0 .
$$

Set

$$
Q=\left\{(t, x) \in[T,+\infty) \times \mathbb{R}, x \leq c_{\lambda+\varepsilon} t+B\right\}
$$

and notice that

$$
\alpha:=\inf _{(t, x) \in \partial Q} u(t, x) \in(0,1) .
$$

Since $f(\alpha)>0$, the weak maximum principle yields $u \geq \alpha$ in $Q$. Consider now any real number $c$ such that $c<c_{\lambda+\varepsilon}$ and assume by contradiction that there exist $\varepsilon_{0}>0$ and a sequence $\left(t_{n}, x_{n}\right)_{n \in \mathbb{N}}$ in $(0,+\infty) \times \mathbb{R}$ such that $t_{n} \rightarrow+\infty$ as $n \rightarrow+\infty$, and $x_{n} \leq c t_{n}$ and $u\left(t_{n}, x_{n}\right) \leq 1-\varepsilon_{0}$ for all $n \in \mathbb{N}$. Set

$$
v_{n}(t, x)=u\left(t+t_{n}, x+x_{n}\right) .
$$

From the Schauder parabolic estimates, the functions $v_{n}$ converge in $\mathcal{C}_{\text {loc }}^{1,2}(\mathbb{R} \times \mathbb{R})$, up to extraction of a subsequence, to a solution $v_{\infty}$ of

$$
\partial_{t} v_{\infty}-\partial_{x x} v_{\infty}=f\left(v_{\infty}\right) \text { in } \mathbb{R} \times \mathbb{R}
$$

such that $0 \leq v_{\infty} \leq 1$. Moreover, for all $(t, x) \in \mathbb{R} \times \mathbb{R}$, there exist $n_{0} \in \mathbb{N}$ large enough so that $\left(t+t_{n}, x+x_{n}\right) \in Q$ for all $n \geq n_{0}$, since $x_{n} \leq c t_{n}$ and $c<c_{\lambda+\varepsilon}$. Thus $v_{\infty} \geq \alpha$ in $\mathbb{R} \times \mathbb{R}$. In particular, it follows from the maximum principle that $v_{\infty}(t, x) \geq \omega\left(t-t_{0}\right)$ for all $t_{0} \in \mathbb{R}$ and for all $(t, x) \in\left[t_{0},+\infty\right) \times \mathbb{R}$, where $\dot{\omega}(t)=f(\omega(t))$ in $[0,+\infty)$ and $\omega(0)=\alpha$. Since $\omega(+\infty)=1$, one concludes, by passing to the limit as $t_{0} \rightarrow-\infty$, that $v_{\infty}(t, x) \geq 1$ for all $(t, x) \in \mathbb{R} \times \mathbb{R}$, which contradicts $v_{\infty}(0,0) \leq 1-\varepsilon_{0}$. Thus,

$$
\inf _{x \leq c t} u(t, x) \rightarrow 1 \text { as } t \rightarrow+\infty \text { for all } c<c_{\lambda+\varepsilon} .
$$

Since $\varepsilon$ was arbitrarily small, one concludes that $w_{*}\left(u_{0}\right) \geq c_{\lambda}$. The proof of Theorem 2.1 is thereby complete.

Proof of Proposition 2.5. It follows from the ideas used in the proof of Theorem 2.1. First, the beginning of the proof of Theorem 2.1 holds with $\varepsilon=0$, since $\Lambda(x)=\lambda$ on $\left[x_{0},+\infty\right)$. Thus, there is $x_{2} \in \mathbb{R}$ such that

$$
\limsup _{t \rightarrow+\infty}\left(\sup _{x \in \mathbb{R}}\left(u(t, x)-U_{c_{\lambda}}\left(x-c_{\lambda} t+x_{2}\right)\right)\right) \leq 0,
$$


which yields the right-hand side inequality in (2.18).

To prove the left-hand side inequality in (2.18), notice that the proof of the lower bound $w_{*}\left(u_{0}\right) \geq c_{\lambda}$ of Theorem 2.1 holds good with $\varepsilon=0$, till the inequality (3.25). Let now $\tau>0$ be any positive real number and remember from the assumption (2.17) and Remark 2.3 that $\eta:=\inf _{x \in \mathbb{R}} \zeta(\tau, x)>0$. On the other hand, $\eta^{\prime}=\inf _{x \leq 0} u(\tau, x)>0$, as noticed in the proof of Theorem 2.1. Finally, it follows from (3.23), (3.24) and (3.25) that

$$
\begin{aligned}
1 \geq u(\tau, x) \geq v_{0}(x):= & \eta^{\prime} \mathbf{1}_{(-\infty, 0]}(x) \\
& +\max \left(0, \kappa \eta e^{-\lambda\left(x-c_{\lambda} \tau\right)}-\kappa A e^{-(\lambda+\theta)\left(x-c_{\lambda} \tau\right)}\right) \mathbf{1}_{(0,+\infty)}(x) \\
\geq & 0
\end{aligned}
$$

for all $x \in \mathbb{R}$. Let $v$ denote the solution of (1.1) with initial condition $v_{0}$. The maximum principle implies that

$$
u(t, x) \geq v(t-\tau, x) \text { for all } t \geq \tau \text { and } x \in \mathbb{R} .
$$

But $v_{0}$ is front-like and $v_{0}(x) \sim \eta^{\prime \prime} e^{-\lambda x}$ as $x \rightarrow+\infty$, with $\eta^{\prime \prime}=\kappa \eta e^{\lambda c_{\lambda} \tau}>0$. It follows then from [24] that there exists $\widetilde{x}_{1} \in \mathbb{R}$ such that $v\left(t, x+c_{\lambda} t\right) \rightarrow U_{c_{\lambda}}\left(x+\widetilde{x}_{1}\right)$ uniformly in $x \in \mathbb{R}$ as $t \rightarrow+\infty$. The inequality (3.26) then gives the left inequality in (2.18) with $x_{1}=\widetilde{x}_{1}+c_{\lambda} \tau$. The proof of Proposition 2.5 is thereby complete.

Remark 3.1 If, in Theorem 2.1, one further imposes the condition (2.17), then the arguments used in the proofs of Theorem 2.1 and Proposition 2.5 imply immediately that, for all $\varepsilon \in\left(0, \lambda^{*}-\lambda\right)$ and for all $t>0$, there is a constant $C_{\varepsilon, t}>0$ such that

$$
u(t, x) \geq C_{\varepsilon, t} \min \left(1, e^{-(\lambda+\varepsilon) x}\right) \text { for all } x \in \mathbb{R} .
$$

The comparison principle and the known results [24] then yield $w_{*}\left(u_{0}\right) \geq c_{\lambda}$. Furthermore, condition (2.15) yields (3.27) as well, at least for $t>0$ large enough. Let us sketch the proof. First, up to replacing $\varepsilon$ with $\varepsilon / 2$, the proof of Theorem 2.1 implies that

$$
u(t, x) \geq \kappa e^{-(\lambda+\varepsilon / 2)\left(x-c_{\lambda+\varepsilon / 2} t\right)}\left(\zeta(t, x-(2 \lambda+\varepsilon) t)-A e^{-\theta\left(x-c_{\lambda+\varepsilon / 2} t\right)}\right)
$$

for all $t>0$ and $x \in \mathbb{R}$, for some $\kappa, A$ and $\theta>0$. On the other hand, (2.15) gives the existence of $c, T$ and $\eta>0$ such that $\zeta(t, c t) \geq \eta$ for all $t \geq T$, that is

$$
\int_{\mathbb{R}} e^{-z^{2}} \bar{\rho}(c t+z \sqrt{4 t}) d z \geq \eta \sqrt{\pi} \text { for all } t \geq T .
$$

Let $D>0$ be such that $\|\bar{\rho}\|_{L^{\infty}(\mathbb{R})} \int_{\mathbb{R} \backslash(-D, D)} e^{-z^{2}} d z \leq(\eta / 2) \sqrt{\pi}$. Since $\bar{\rho} \geq 0$, it follows that, for all $x \geq c T(>0)$, there holds

$$
\int_{-D}^{D} \bar{\rho}(x+z \sqrt{4 x / c}) d z \geq \int_{-D}^{D} e^{-z^{2}} \bar{\rho}(x+z \sqrt{4 x / c}) d z \geq \frac{\eta}{2} \sqrt{\pi} .
$$

Fix now any $\beta \in(0, \min (\varepsilon / 2, \theta))$ and any $t>D^{2} /(\beta c)$. For all $x \geq c T$, one has

$$
\begin{aligned}
\zeta(t, x-(2 \lambda+\varepsilon) t) & =\frac{1}{\sqrt{\pi}} \int_{\mathbb{R}} e^{-z^{2}} \bar{\rho}(x-(2 \lambda+\varepsilon) t+z \sqrt{4 t}) d z \\
& =\sqrt{\frac{x}{\pi c t}} \int_{\mathbb{R}} e^{-((\lambda+\varepsilon / 2) \sqrt{t}+y \sqrt{x /(c t)})^{2}} \bar{\rho}(x+y \sqrt{4 x / c}) d y \\
& \geq \frac{\eta}{2} \sqrt{\frac{x}{c t}} e^{-((\lambda+\varepsilon / 2) \sqrt{t}+D \sqrt{x /(c t)})^{2}}
\end{aligned}
$$


because of (3.29) and $\bar{\rho} \geq 0$. Thus,

$$
\lim _{x \rightarrow+\infty} e^{\beta x} \zeta(t, x-(2 \lambda+\varepsilon) t)=+\infty \text { for } t>\frac{D^{2}}{\beta c} .
$$

Since $u(t, \cdot)$ is positive and continuous, since $\liminf _{x \rightarrow-\infty} u(t, x)>0$ and since $0<\beta<\min (\varepsilon / 2, \theta)$, it follows then that (3.27) holds for some $C_{\varepsilon, t}>0$, owing to (3.28), whence $w_{*}\left(u_{0}\right) \geq c_{\lambda+\varepsilon}$ for all $\varepsilon>0$ small enough. Summing up, (2.15) and (2.17) give alternate conditions, which are expressed directly in terms of simple properties of some solutions of the heat equation, to apply the known results [24] and get $w_{*}\left(u_{0}\right) \geq c_{\lambda}$. In the proof of Theorem 2.1 , this inequality was shown with another method, which is based directly on comparison principles and Liouville type results and which does not use [24]. Lastly, notice that the conditions (2.15) or (2.17) are not equivalent to (3.27). For instance, if $\rho(x)>0, \rho(x) \rightarrow 0$ and $|\ln (\rho(x))|=o(x)$ as $x \rightarrow+\infty$, then $u_{0}$ can be written as $u_{0}(x)=e^{-(\lambda+\varepsilon) x} \widetilde{\rho}_{\varepsilon}(x)$ for all $\varepsilon>0$ and a.e. $x>x_{0}$ where $\widetilde{\rho}_{\varepsilon}(x)=e^{(\lambda+\varepsilon-\Lambda(x))(x)} \rho(x) \rightarrow+\infty$ as $x \rightarrow+\infty$; thus, the arguments above yield (3.27); but neither (2.17) nor even (2.15) holds, as already observed in Section 2 after Remark 2.3.

Proof of Corollary 2.4. Consider the solution $\zeta$ of (2.14), where the function $\rho$ is assumed to be asymptotically positive in average at $+\infty$, that is there are $\alpha>0, \beta>0$ and $A>0$ such that

$$
h^{-1} \int_{x}^{x+h} \rho(z) d z \geq \alpha \text { for all } x \geq A \text { and } h \geq \beta \sqrt{x} .
$$

Let us now check that $\liminf \operatorname{int}_{t \rightarrow+\infty} \zeta(t, c t)>0$ for all $c>0$, which will then yield $(2.15)$ and the desired conclusion from Theorem 2.1. To do so, set

$$
R_{x}(y)=\int_{x}^{y} \bar{\rho}(z) d x
$$

for all $(x, y) \in \mathbb{R}^{2}$, and fix a speed $c>0$. For all $t>0$, one has

$$
\zeta(t, c t)=\frac{1}{\sqrt{4 \pi t}} \int_{\mathbb{R}} e^{-\frac{y^{2}}{4 t}} \bar{\rho}(c t-y) d y=\frac{1}{\sqrt{4 \pi t}} \int_{\mathbb{R}} \frac{y}{2 t} e^{-\frac{y^{2}}{4 t}} R_{c t}(c t+y) d y
$$

after integrating by parts (notice that $\left|R_{c t}(c t+y)\right|=O(|y|)$ as $|y| \rightarrow+\infty$ for every $t>0$ ). Since $\bar{\rho} \geq 0$, one has $y R_{c t}(c t+y) \geq 0$ for all $t>0$ and $y \in \mathbb{R}$, whence, for all $t \geq \max \left(A, x_{0}\right) / c$,

$$
\zeta(t, c t) \geq \frac{1}{\sqrt{4 \pi t}} \int_{\beta \sqrt{c t}}^{+\infty} \frac{y^{2}}{c t} e^{-\frac{y^{2}}{4 t}} \alpha d y=\frac{4 \alpha}{c \sqrt{\pi}} \int_{\beta \sqrt{c / 4}}^{+\infty} z^{2} e^{-z^{2}} d z .
$$

Since the right-hand side is positive and independent of $t$, the proof is thereby complete.

Proof of Corollary 2.6. The same kind of argument as in the proof of Theorem 2.1 implies that for all $\varepsilon \in\left(0, \lambda^{*}\right)$, one has

$$
w_{*}\left(u_{0}\right) \geq \varepsilon+\frac{f^{\prime}(0)}{\varepsilon} .
$$

We get the conclusion by letting $\varepsilon \rightarrow 0^{+}$. 


\section{Complex dynamics and intervals of spreading speeds}

This section is devoted to the proof of Theorem 2.7. That is, we construct explicit examples of front-like initial conditions $u_{0}$ for which the minimal and maximal spreading speeds $w_{*}\left(u_{0}\right)$ and $w^{*}\left(u_{0}\right)$ are any two given strictly ordered numbers between $2 \sqrt{M_{f}}$ and $+\infty$, where $M_{f}=\max _{s \in[0,1]} f^{\prime}(s)$. The constructed functions $u_{0}$ oscillate at $+\infty$ between two exponentially decaying functions, with different exponential rates. The intervals of oscillation are larger and larger. They are chosen in such a way that, during some suitable time-intervals and on some space-intervals, the Gaussian estimates of the difference between the solution $u$ and two approximated fronts is negligible.

Proof of Theorem 2.7. Let $\gamma_{1}<\gamma_{2}$ be given in the closed interval $\left[2 \sqrt{M_{f}},+\infty\right] \subset\left[c^{*},+\infty\right]$. If $\gamma_{1}>c^{*}$, let $\lambda_{1} \in\left(0, \lambda^{*}\right)$ be such that $c_{\lambda_{1}}=\gamma_{1}$, that is

$$
\lambda_{1}=\frac{\gamma_{1}-\sqrt{\gamma_{1}^{2}-4 f^{\prime}(0)}}{2}
$$

If $\gamma_{1}=c^{*}$, set $\lambda_{1}=\lambda^{*}$. Let also $\lambda_{2}$ be the unique real number in $\left[0, \lambda^{*}\right)$ such that $c_{\lambda_{2}}=\gamma_{2}$ (with the convention that $c_{0}=+\infty$ ). In all cases, there holds

$$
0 \leq \lambda_{2}<\lambda_{1} \leq \lambda^{*}
$$

Let $\left(\lambda_{2, n}\right)_{n \in \mathbb{N}}$ be the sequence defined by

$$
\forall n \in \mathbb{N}, \quad \begin{cases}\lambda_{2, n}=\lambda_{2} & \text { if } \lambda_{2}>0, \\ \lambda_{2, n}=\frac{\lambda_{1}}{n+2} & \text { if } \lambda_{2}=0,\end{cases}
$$

and let $\left(x_{n}\right)_{n \in \mathbb{N}}$ and $\left(y_{n}\right)_{n \in \mathbb{N}}$ be any two increasing sequences of positive real numbers such that

$$
0<x_{n}<y_{n}<\frac{\lambda_{1}}{\lambda_{2, n}} y_{n}<x_{n+1}-1<x_{n+1} \text { for all } n \in \mathbb{N}
$$

and

$$
\lim _{n \rightarrow+\infty} \frac{y_{n}}{x_{n}}=\lim _{n \rightarrow+\infty} \frac{x_{n+1}}{\left(\lambda_{1} / \lambda_{2, n}\right) y_{n}}=+\infty .
$$

Typical examples are $x_{n}=\left(2 n+n_{0}\right)$ ! and $y_{n}=\left(2 n+1+n_{0}\right)$ ! if $\lambda_{2}>0\left(\right.$ resp. $x_{n}=\left(\left(2 n+n_{0}\right) !\right)^{2}$ and $y_{n}=\left(\left(2 n+1+n_{0}\right) \text { ! }\right)^{2}$ if $\left.\lambda_{2}=0\right)$, for some large enough integer $n_{0}$.

Given any such sequences $\left(x_{n}\right)_{n \in \mathbb{N}}$ and $\left(y_{n}\right)_{n \in \mathbb{N}}$, we define the function $u_{0}$ as follows:

$$
u_{0}(x)= \begin{cases}\min \left(1, e^{-\lambda_{1} x}\right) & \text { if } x<x_{0}, \\ e^{-\lambda_{1} x} & \text { if } x_{n} \leq x<y_{n}, \\ e^{-\lambda_{1} y_{n}} & \text { if } y_{n} \leq x<\frac{\lambda_{1}}{\lambda_{2, n}} y_{n}, \\ e^{-\lambda_{2, n} x} & \text { if } \frac{\lambda_{1}}{\lambda_{2, n}} y_{n} \leq x<x_{n+1}-1, \\ e^{-\lambda_{1} x_{n+1}} & \text { if } x_{n+1}-1 \leq x<x_{n+1}, \\ +\left(e^{-\lambda_{2, n}\left(x_{n+1}-1\right)}-e^{-\lambda_{1} x_{n+1}}\right)\left(x_{n+1}-x\right)\end{cases}
$$




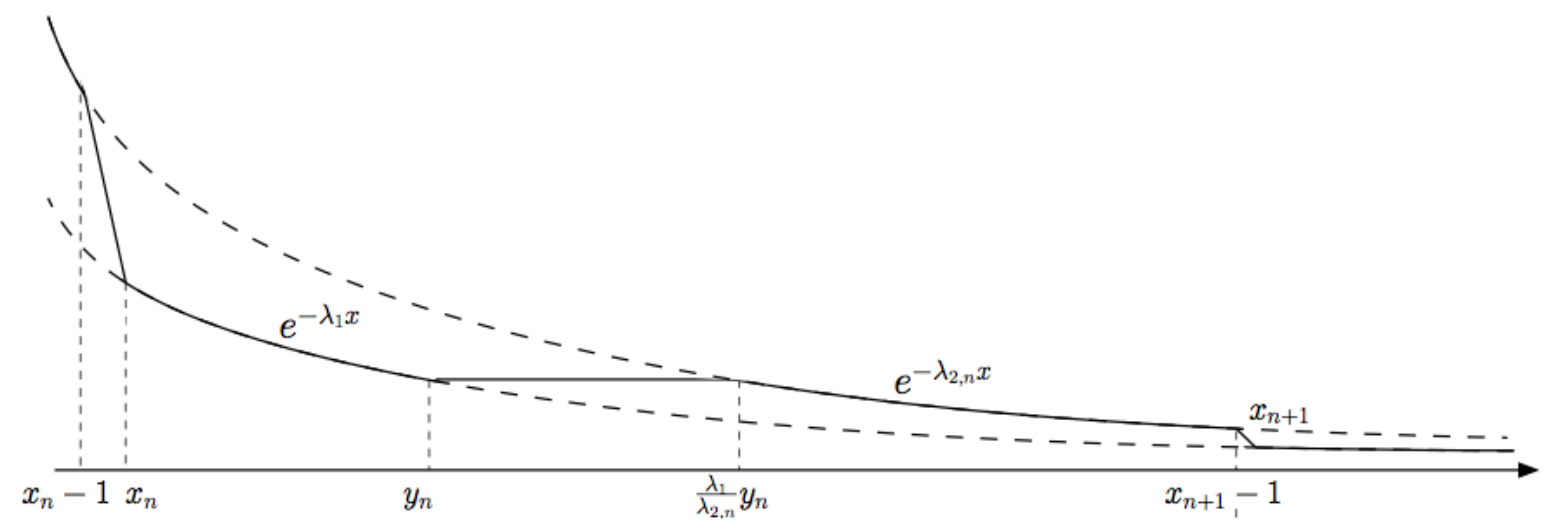

see the joint figure. The function $u_{0}$ is thus continuous, front-like in the sense of Definition 1.1, non-increasing in $\mathbb{R}$, and $u_{0}(-\infty)=1$. Let $u$ be the solution of (1.1) with the initial condition $u_{0}$ and let us check that the conclusion of Theorem 2.7 holds with this $u_{0}$.

The function $u_{0}$ oscillates between $e^{-\lambda_{1} x}$ and $e^{-\lambda_{2} x}$ (or $e^{-\lambda_{2, n} x}$ if $\lambda_{2}=0$ ) as $x \rightarrow+\infty$. It is also glued between these two exponentially decaying functions between $y_{n}$ and $\left(\lambda_{1} / \lambda_{2, n}\right) y_{n}$ and between $x_{n+1}-1$ and $x_{n+1}$ in such a way that it is nonincreasing. This monotonicity property will be inherited at all positive times, which reduces the level sets $E_{m}(t)$ to singletons (and will then help in the calculations of their positions). Namely, the strong maximum principle implies that, for every $t>0$, the function $u(t, \cdot)$ is decreasing on $\mathbb{R}$, and $u(t,-\infty)=1, u(t,+\infty)=0$. Therefore, for every $t>0$ and $m \in(0,1)$, the level set $E_{m}(t)$ reduces to a singleton

$$
E_{m}(t)=\left\{x_{m}(t)\right\}
$$

Furthermore, the functions $t \mapsto x_{m}(t)$ are all (at least) of class $C^{1}$ on $(0,+\infty)$ from the implicit function theorem.

Since $u_{0}$ is front-like and

$$
e^{-\lambda_{1} x} \leq u_{0}(x) \leq e^{-\lambda_{2} x} \text { for all } x \geq 0
$$

it follows from the maximum principle, together with [24] (or Corollary 2.2) and the general comparisons (1.6), that

$$
\gamma_{1} \leq w_{*}\left(u_{0}\right) \leq w^{*}\left(u_{0}\right) \leq \gamma_{2} .
$$

It also follows from the definitions of the spreading speeds that, for every $m \in(0,1)$,

$$
\gamma_{1} \leq w_{*}\left(u_{0}\right) \leq \liminf _{t \rightarrow+\infty} \frac{x_{m}(t)}{t} \leq \limsup _{t \rightarrow+\infty} \frac{x_{m}(t)}{t} \leq w^{*}\left(u_{0}\right) \leq \gamma_{2}
$$

Next, let $\underline{u}_{0}$ and $\bar{u}_{0}$ be the two functions defined on $\mathbb{R}$ by

$$
\left\{\begin{array}{l}
\underline{u}_{0}(x)= \begin{cases}1 & \text { if } x<0 \\
e^{-\lambda_{1} x} & \text { if } x \geq 0\end{cases} \\
\bar{u}_{0}(x)= \begin{cases}1 & \text { if } x<0 \\
e^{-\lambda_{2, n} x} & \text { if } x_{n} \leq x<x_{n+1}\end{cases}
\end{array}\right.
$$


Observe that, if $\lambda_{2}>0$, then $\bar{u}_{0}(x)=e^{-\lambda_{2} x}$ for all $x \geq 0$. The function $\underline{u}_{0}$ is obviously frontlike, as is the function $\bar{u}_{0}$ if $\lambda_{2}>0$. If $\lambda_{2}=0$, then $\lambda_{2, n}=\lambda_{1} /(n+2)$, whence $\lambda_{2, n} x_{n} \rightarrow+\infty$ as $n \rightarrow+\infty$ (since $x_{n+1} / x_{n} \rightarrow+\infty$ ) and $\bar{u}_{0}(x) \rightarrow 0$ as $x \rightarrow+\infty$. In other words, the function $\bar{u}_{0}$ is front-like whenever $\lambda_{2}$ is positive or 0 . Let $\underline{u}$ and $\bar{u}$ be the solutions of (1.1) with initial conditions $\underline{u}_{0}$ and $\bar{u}_{0}$. Since $0 \leq \underline{u}_{0} \leq u_{0} \leq \bar{u}_{0} \leq 1$ on $\mathbb{R}$, the maximum principle yields

$$
0 \leq \underline{u}(t, x) \leq u(t, x) \leq \bar{u}(t, x) \leq 1 \text { for all } t \geq 0 \text { and } x \in \mathbb{R} .
$$

Furthermore, as already recalled in Section 1, it follows from Uchiyama [24] that

$$
\sup _{x \in \mathbb{R}}\left|\underline{u}(t, x)-U_{\gamma_{1}}\left(x-\gamma_{1} t+m_{1}(t)\right)\right| \rightarrow 0 \text { as } t \rightarrow+\infty
$$

where $m_{1}(t) / t \rightarrow 0$ as $t \rightarrow+\infty$ (moreover, if $\gamma_{1}>c^{*}$, then $m_{1}(t)$ can be chosen to be a constant real number $x_{1}$ in the above formula). Similarly, if $\gamma_{2}<+\infty$ (that is, $\lambda_{2}>0$ ), then there exists $x_{2} \in \mathbb{R}$ such that

$$
\sup _{x \in \mathbb{R}}\left|\bar{u}(t, x)-U_{\gamma_{2}}\left(x-\gamma_{2} t+x_{2}\right)\right| \rightarrow 0 \text { as } t \rightarrow+\infty \text {. }
$$

Let us now prove that these two approximated travelling fronts $U_{\gamma_{1}}\left(x-\gamma_{1} t+m_{1}(t)\right)$ and $U_{\gamma_{2}}\left(x-\gamma_{2} t+x_{2}\right)$ (if $\gamma_{2}<+\infty$ ) are closer and closer to $u$ on some larger and larger space-intervals during some larger and larger time-intervals. That will be sufficient to get the conclusion of Theorem 2.7 (at least if $\gamma_{2}<+\infty$, the case $\gamma_{2}=+\infty$ is analysed separately).

To do so, denote

$$
v=u-\underline{u} \geq 0 \text { and } w=\bar{u}-u \geq 0 \text { on }[0,+\infty) \times \mathbb{R} .
$$

Choose any sequences $\left(t_{n}\right)_{n \in \mathbb{N}}$ and $\left(t_{n}^{\prime}\right)_{n \in \mathbb{N}}$ of positive real numbers such that

$$
x_{n}<t_{n} \leq t_{n}^{\prime}<y_{n} \text { for all } n \in \mathbb{N} \text { and } \lim _{n \rightarrow+\infty} \frac{t_{n}}{x_{n}}=\lim _{n \rightarrow+\infty} \frac{y_{n}}{t_{n}^{\prime}}=+\infty .
$$

Such sequences exist since $y_{n} / x_{n} \rightarrow+\infty$ as $n \rightarrow+\infty$. For instance, a particular choice is: $t_{n}=x_{n}^{1-\theta} y_{n}^{\theta}$ and $t_{n}^{\prime}=x_{n}^{1-\theta^{\prime}} y_{n}^{\theta^{\prime}}$ with $0<\theta \leq \theta^{\prime}<1$. We now claim that

$$
\max _{t \in\left[t_{n}, t_{n}^{\prime}\right]}\left(\max _{x \in\left[\left(2 \sqrt{M_{f}}+\varepsilon\right) t, \gamma t\right]} v(t, x)\right) \rightarrow 0 \text { as } n \rightarrow+\infty
$$

for any two positive real numbers $\varepsilon$ and $\gamma$ such that $2 \sqrt{M_{f}}+\varepsilon \leq \gamma$. This property will imply that the solution $u$ is close to $\underline{u}$ and then to the approximated front $U_{\gamma_{1}}\left(x-\gamma_{1} t+m_{1}(t)\right)$ on sequences of time-intervals $\left[t_{n}, t_{n}^{\prime}\right]$ and on some space-intervals, provided that the ratio between the position and the time belongs to $\left[2 \sqrt{M_{f}}+\varepsilon, \gamma\right]$. Since $\varepsilon>0$ can be arbitrarily small, the equality $w_{*}\left(u_{0}\right)=\gamma_{1}$ will follow.

In order to prove (4.35), let $\varepsilon>0$ and $\gamma>0$ be as above and denote, for all $n \in \mathbb{N}$,

$$
E_{n}^{\varepsilon, \gamma}=\left\{(t, x) \in(0,+\infty) \times \mathbb{R}, t_{n} \leq t \leq t_{n}^{\prime},\left(2 \sqrt{M_{f}}+\varepsilon\right) t \leq x \leq \gamma t\right\}
$$


Observe that

$$
0 \leq v(0, x)=u_{0}(x)-\underline{u}_{0}(x) \leq \sum_{n \in \mathbb{N}} \mathbf{1}_{\left[y_{n}, x_{n+1}\right]}(x) \text { for all } x \in \mathbb{R}
$$

and that

$$
\partial_{t} v(t, x)-\partial_{x x} v(t, x)=f(u(t, x))-f(\underline{u}(t, x)) \leq M_{f} v(t, x) \text { for all }(t, x) \in(0,+\infty) \times \mathbb{R},
$$

owing to the definition of $M_{f}=\max _{s \in[0,1]} f^{\prime}(s)$ and the nonnegativity of $v$. The maximum principle implies then that, for all $(t, x) \in(0,+\infty) \times \mathbb{R}$,

$$
0 \leq v(t, x) \leq \frac{e^{M_{f} t}}{\sqrt{4 \pi t}} \sum_{n \in \mathbb{N}} \int_{y_{n}}^{x_{n+1}} e^{-\frac{(x-y)^{2}}{4 t}} d y .
$$

Then, choose $n_{1} \in \mathbb{N}$ such that $x_{n} \leq\left(2 \sqrt{M_{f}}+\varepsilon\right) t_{n} \leq \gamma t_{n}^{\prime} \leq y_{n}$ for all $n \geq n_{1}$. For any $n \geq n_{1}$ and $(t, x) \in E_{n}^{\varepsilon, \gamma}$, one then has

$$
x_{n} \leq\left(2 \sqrt{M_{f}}+\varepsilon\right) t_{n} \leq\left(2 \sqrt{M_{f}}+\varepsilon\right) t \leq x \leq \gamma t \leq \gamma t_{n}^{\prime} \leq y_{n},
$$

whence

$$
\begin{aligned}
0 \leq v(t, x) & \leq \frac{e^{M_{f} t}}{\sqrt{4 \pi t}} \times\left(\int_{-\infty}^{x_{n}} e^{-\frac{(x-y)^{2}}{4 t}} d y+\int_{y_{n}}^{+\infty} e^{-\frac{(x-y)^{2}}{4 t}} d y\right) \\
& =\frac{e^{M_{f} t}}{\sqrt{\pi}} \int_{-\infty}^{\frac{x_{n}-x}{\sqrt{4 t}}} e^{-z^{2}} d z+\frac{e^{M_{f} t}}{\sqrt{\pi}} \int_{\frac{y_{n}-x}{\sqrt{4 t}}}^{+\infty} e^{-z^{2}} d z,
\end{aligned}
$$

from (4.36). But

$$
\begin{aligned}
\frac{x_{n}-x}{\sqrt{4 t}} \leq \frac{x_{n}-\left(2 \sqrt{M_{f}}+\varepsilon\right) t}{\sqrt{4 t}} & =-\sqrt{t} \times\left(\sqrt{M_{f}}+\frac{\varepsilon}{2}-\frac{x_{n}}{2 t}\right) \\
& \leq-\sqrt{t} \times\left(\sqrt{M_{f}}+\frac{\varepsilon}{2}-\frac{x_{n}}{2 t_{n}}\right)
\end{aligned}
$$

and $x_{n} / t_{n} \rightarrow 0$ as $n \rightarrow+\infty$. Therefore, there exists $n_{2} \geq n_{1}$ such that

$$
\frac{x_{n}-x}{\sqrt{4 t}} \leq-\sqrt{M_{f} t} \leq-\sqrt{M_{f} t_{n}}<0 \text { for all } n \geq n_{2} \text { and }(t, x) \in E_{n}^{\varepsilon, \gamma} .
$$

On the other hand, $\int_{A}^{+\infty} e^{-z^{2}} d z \leq e^{-A^{2}} /(2 A)$ for all $A>0$. Therefore,

$$
\begin{aligned}
\frac{e^{M_{f} t}}{\sqrt{\pi}} \int_{-\infty}^{\frac{x_{n}-x}{\sqrt{4 t}}} e^{-z^{2}} d z & \leq \frac{e^{M_{f} t}}{\sqrt{\pi}} \times \int_{-\infty}^{-\sqrt{M_{f} t}} e^{-z^{2}} d z \\
& \leq \frac{e^{M_{f} t}}{\sqrt{\pi}} \times \frac{e^{-M_{f} t}}{\sqrt{4 M_{f} t}} \leq \frac{1}{\sqrt{4 \pi M_{f} t_{n}}}
\end{aligned}
$$

for all $n \geq n_{2}$ and $(t, x) \in E_{n}^{\varepsilon, \gamma}$. As far as the second term in the right-hand side of (4.37) is concerned, one knows that

$$
\frac{y_{n}-x}{\sqrt{4 t}} \geq \frac{y_{n}-\gamma t_{n}^{\prime}}{2 \sqrt{t_{n}^{\prime}}} \geq \frac{y_{n}}{4 \sqrt{t_{n}^{\prime}}} \text { for all } n \text { large enough, }
$$


since $y_{n} / t_{n}^{\prime} \rightarrow+\infty$ as $n \rightarrow+\infty$. Thus, there exists $n_{3} \geq n_{2}$ such that

$$
\frac{e^{M_{f} t}}{\sqrt{\pi}} \int_{\frac{y_{n}-x}{\sqrt{4 t}}}^{+\infty} e^{-z^{2}} d z \leq \frac{e^{M_{f} t_{n}^{\prime}}}{\sqrt{\pi}} \int_{\frac{y_{n}}{4 \sqrt{t_{n}^{\prime}}}}^{+\infty} e^{-z^{2}} d z \leq \frac{e^{M_{f} t_{n}^{\prime}}}{\sqrt{\pi}} \times e^{-\frac{y_{n}^{2}}{16 t_{n}^{\prime}}} \times \frac{2 \sqrt{t_{n}^{\prime}}}{y_{n}}
$$

for all $n \geq n_{3}$ and $(t, x) \in E_{n}^{\varepsilon, \gamma}$. Combining (4.37), (4.38) and (4.39), one infers that

$$
\max _{(t, x) \in E_{n}^{\varepsilon, \gamma}} v(t, x) \leq \frac{1}{\sqrt{4 \pi M_{f} t_{n}}}+\frac{e^{M_{f} t_{n}^{\prime}}}{\sqrt{\pi}} \times e^{-\frac{y_{n}^{2}}{16 t_{n}^{\prime}}} \times \frac{2 \sqrt{t_{n}^{\prime}}}{y_{n}}
$$

for all $n \geq n_{3}$. But the right-hand side converges to 0 as $n \rightarrow+\infty$, since $t_{n}, y_{n} / t_{n}^{\prime}$ and $y_{n} / \sqrt{t_{n}^{\prime}}$ all converge to $+\infty$ as $n \rightarrow+\infty$. This provides (4.35).

Putting together $(4.33),(4.35)$ and the fact that $U_{\gamma_{1}}(+\infty)=0$, it follows that, for all $A \in \mathbb{R}$ and $\left(2 \sqrt{M_{f}} \leq\right) \gamma_{1}<c<\gamma$, there holds

$$
\max _{t \in\left[t_{n}, t_{n}^{\prime}\right]}\left(\max _{x \in[c t+A, \gamma t]} u(t, x)\right) \rightarrow 0 \text { as } n \rightarrow+\infty
$$

In particular,

$$
\max _{t \in\left[t_{n}, t_{n}^{\prime}\right]} u(t, c t+x) \rightarrow 0 \text { as } n \rightarrow+\infty \text { for all } c>\gamma_{1} \text { and } x \in \mathbb{R} .
$$

Since $u(t, \cdot)$ is decreasing for all $t>0$, one actually gets that

$$
\max _{t \in\left[t_{n}, t_{n}^{\prime}\right]}\left(\max _{x \in[c t+A,+\infty)} u(t, x)\right) \rightarrow 0 \text { as } n \rightarrow+\infty
$$

for all $A \in \mathbb{R}$ and $c>\gamma_{1}$. Therefore, for all $m \in(0,1), \liminf _{t \rightarrow+\infty} x_{m}(t) / t \leq \gamma_{1}$ and eventually

$$
\liminf _{t \rightarrow+\infty} \frac{x_{m}(t)}{t}=\gamma_{1}
$$

because of (4.31). Furthermore, $w_{*}\left(u_{0}\right) \leq \gamma_{1}$, and (4.31) also yields the equality $w_{*}\left(u_{0}\right)=\gamma_{1}$.

Let us now prove that $w^{*}\left(u_{0}\right)=\gamma_{2}$ and $\lim \sup _{t \rightarrow+\infty} x_{m}(t) / t=\gamma_{2}$ for all $m \in(0,1)$. Remember the definition of $\bar{u}_{0}$ in (4.32), and that $w=\bar{u}-u \geq 0$ in $[0,+\infty) \times \mathbb{R}$. Choose any sequences $\left(\tau_{n}\right)_{n \in \mathbb{N}}$ and $\left(\tau_{n}^{\prime}\right)_{n \in \mathbb{N}}$ of positive real numbers such that

$$
\left\{\begin{array}{l}
\frac{\lambda_{1}}{\lambda_{2, n}} y_{n}<\tau_{n} \leq \tau_{n}^{\prime}<x_{n+1}-1 \text { for all } n \in \mathbb{N} \\
\lim _{n \rightarrow+\infty} \frac{\tau_{n}}{\left(\lambda_{1} / \lambda_{2, n}\right) y_{n}}=\lim _{n \rightarrow+\infty} \frac{x_{n+1}}{\tau_{n}^{\prime}}=+\infty .
\end{array}\right.
$$

Such sequences exist because of (4.30). Since $w(0, \cdot)=\bar{u}_{0}-u_{0}=0$ on all the intervals $\left[\left(\lambda_{1} / \lambda_{2, n}\right) y_{n}, x_{n+1}-1\right]$ for all $n \in \mathbb{N}$, the same arguments as for the function $v$ imply that

$$
\max _{t \in\left[\tau_{n}, \tau_{n}^{\prime}\right]}\left(\max _{x \in\left[\left(2 \sqrt{M_{f}}+\varepsilon\right) t, \gamma t\right]} w(t, x)\right) \rightarrow 0 \text { as } n \rightarrow+\infty
$$

for any two positive real numbers $\varepsilon$ and $\gamma$ such that $2 \sqrt{M_{f}}+\varepsilon \leq \gamma$. 
Consider first the case $\gamma_{2}<+\infty$ (that is, $\lambda_{2}>0$ ). It follows then from (4.34), (4.42) and $U_{\gamma_{2}}(-\infty)=1$ that, for all $A \in \mathbb{R}$ and for all $2 \sqrt{M_{f}}<c^{\prime}<c<\gamma_{2}$, there holds

$$
\min _{t \in\left[\tau_{n}, \tau_{n}^{\prime}\right]}\left(\min _{x \in\left[c^{\prime} t, c t+A\right]} u(t, x)\right) \rightarrow 1 \text { as } n \rightarrow+\infty .
$$

Since $u(t, \cdot)$ is decreasing for all $t>0$, one actually gets that

$$
\min _{t \in\left[\tau_{n}, \tau_{n}^{\prime}\right]}\left(\min _{x \in(-\infty, c t+A)} u(t, x)\right) \rightarrow 1 \text { as } n \rightarrow+\infty
$$

for all $A \in \mathbb{R}$ and $c<\gamma_{2}$. In particular,

$$
\min _{t \in\left[\tau_{n}, \tau_{n}^{\prime}\right]} u(t, c t+x) \rightarrow 1 \text { as } n \rightarrow+\infty \text { for all } c<\gamma_{2} \text { and } x \in \mathbb{R} .
$$

Furthermore, for all $m \in(0,1)$, $\lim \sup _{t \rightarrow+\infty} x_{m}(t) / t \geq \gamma_{2}$, whence $\lim \sup _{t \rightarrow+\infty} x_{m}(t) / t=\gamma_{2}$ because of (4.31). Eventually, $w^{*}\left(u_{0}\right) \geq \gamma_{2}$, and (4.31) yields $w^{*}\left(u_{0}\right)=\gamma_{2}$.

Lastly, consider the case $\gamma_{2}=+\infty$ (that is, $\lambda_{2}=0$ ). Let $\eta$ be any real number in the interval $\left(0, \lambda^{*}\right)$. Let $n_{\eta} \in \mathbb{N}$ be such that $0<\lambda_{2, n}<\eta$ for all $n \geq n_{\eta}$. Define the function $u_{0}^{\eta}: \mathbb{R} \rightarrow[0,1]$ by

$$
u_{0}^{\eta}(x)= \begin{cases}1 \quad & \text { if } x<0, \\ 0 & \text { if } 0 \leq x<\frac{\lambda_{1}}{\lambda_{2, n_{\eta}}} y_{n_{\eta}}, \\ e^{-\eta x} & \text { if } \frac{\lambda_{1}}{\lambda_{2, n}} y_{n} \leq x<x_{n+1}-1 \text { with } n \geq n_{\eta}, \\ 0 & \text { if } x_{n+1}-1 \leq x<\frac{\lambda_{1}}{\lambda_{2, n+1}} y_{n+1} \text { with } n \geq n_{\eta} .\end{cases}
$$

From the choice of $n_{\eta}$ and $u_{0}$, one has $u_{0} \geq u_{0}^{\eta}$ on $\mathbb{R}$, whence

$$
u(t, x) \geq u^{\eta}(t, x) \text { for all } t>0 \text { and } x \in \mathbb{R}
$$

from the maximum principle, where $u^{\eta}$ denotes the solution of the equation (1.1) with initial condition $u_{0}^{\eta}$. Define now

$$
\bar{u}_{0}^{\eta}(x)= \begin{cases}1 & \text { if } x<\frac{\lambda_{1}}{\lambda_{2, n_{\eta}}} y_{n_{\eta}}, \\ e^{-\eta x} & \text { if } x \geq \frac{\lambda_{1}}{\lambda_{2, n_{\eta}}} y_{n_{\eta}}\end{cases}
$$

and let $\bar{u}^{\eta}$ be the solution of problem (1.1) with initial condition $\bar{u}_{0}^{\eta}$. Since $\bar{u}_{0}^{\eta} \geq u_{0}^{\eta}$ on $\mathbb{R}$, the maximum principle yields

$$
w^{\eta}(t, x)=\bar{u}^{\eta}(t, x)-u^{\eta}(t, x) \geq 0 \text { for all } t>0 \text { and } x \in \mathbb{R} .
$$

Furthermore, since $\bar{u}_{0}^{\eta}=u_{0}^{\eta}$ on the intervals $\left[\left(\lambda_{1} / \lambda_{2, n}\right) y_{n}, x_{n+1}-1\right)$ for all $n \geq n_{\eta}$, the same arguments as above imply that

$$
\max _{t \in\left[\tau_{n}, \tau_{n}^{\prime}\right]}\left(\max _{x \in\left[\left(2 \sqrt{M_{f}}+\varepsilon\right) t, \gamma t\right]} w^{\eta}(t, x)\right) \rightarrow 0 \text { as } n \rightarrow+\infty
$$


for all $\varepsilon>0$ and $\gamma<+\infty$ such that $2 \sqrt{M_{f}}+\varepsilon \leq \gamma$. On the other hand, because of Uchiyama [24], there exists $x_{\eta} \in \mathbb{R}$ such that

$$
\sup _{x \in \mathbb{R}}\left|\bar{u}^{\eta}(t, x)-U_{c_{\eta}}\left(x-c_{\eta} t+x_{\eta}\right)\right| \rightarrow 0 \text { as } t \rightarrow+\infty,
$$

where $c_{\eta}=\eta+f^{\prime}(0) / \eta$. Since $U_{c_{\eta}}(-\infty)=1$, one then infers that

$$
\min _{t \in\left[\tau_{n}, \tau_{n}^{\prime}\right]}\left(\min _{x \in\left[c^{\prime} t, c t+A\right]} u^{\eta}(t, x)\right) \rightarrow 1 \text { as } n \rightarrow+\infty
$$

for all $A \in \mathbb{R}$ and $2 \sqrt{M_{f}}<c^{\prime}<c<c_{\eta}$. Remember now that $u \geq u^{\eta}$ from (4.44) and that $u(t, \cdot)$ is decreasing for all $t>0$. Therefore,

$$
\min _{t \in\left[\tau_{n}, \tau_{n}^{\prime}\right]}\left(\min _{x \in(-\infty, c t+A]} u(t, x)\right) \rightarrow 1 \text { as } n \rightarrow+\infty
$$

for all $A \in \mathbb{R}$ and $c<c_{\eta}$. Since $\eta \in\left(0, \lambda^{*}\right)$ can be chosen arbitrarily small and $c_{\eta} \rightarrow+\infty$ as $\eta \rightarrow 0^{+}$, it follows that (4.45) holds for all $c \in \mathbb{R}$ and $A \in \mathbb{R}$. In particular,

$$
\min _{t \in\left[\tau_{n}, \tau_{n}^{\prime}\right]} u(t, c t+x) \rightarrow 1 \text { as } n \rightarrow+\infty \text { for all } c<+\infty \text { and } x \in \mathbb{R} .
$$

Moreover, $\lim \sup _{t \rightarrow+\infty} x_{m}(t) / t=+\infty$ for all $m \in(0,1)$ and $w^{*}\left(u_{0}\right)=+\infty$.

As a conclusion, whenever $\gamma_{2}$ is finite or $+\infty$, there always holds $w^{*}\left(u_{0}\right)=\gamma_{2}$, and $\limsup _{t \rightarrow+\infty} x_{m}(t) / t=\gamma_{2}$ for all $m \in(0,1)$. Because of (4.41), one concludes that, for all $m \in(0,1)$, the $\omega$-limit set of the (continuous on $(0,+\infty))$ function $t \mapsto x_{m}(t) / t$ is equal to the whole interval $\left[\gamma_{1}, \gamma_{2}\right]$. Lastly, the limits (4.40), (4.43) and (4.46) imply that, for all $c \in\left(\gamma_{1}, \gamma_{2}\right)$ and $x \in \mathbb{R}$, the $\omega$-limit set of the (continuous on $\left.(0,+\infty)\right)$ function $t \mapsto u(t, c t+x)$ is equal to the whole interval $[0,1]$. The proof of Theorem 2.7 is thereby complete.

Remark 4.1 If $\gamma_{1}>c^{*}$, then the quantity $m_{1}(t)$ appearing in (4.33) can be chosen to be a constant real number $x_{1}$. Together with the inequality $u \geq \underline{u}$ and formula (4.43) applied with $c=\gamma_{1}<\gamma_{2}$, it follows that, for each $x \in \mathbb{R}$, the $\omega$-limit set of the function $t \mapsto u\left(t, \gamma_{1} t+x\right)$ is equal to the interval $\left[U_{\gamma_{1}}\left(x+x_{1}\right), 1\right]$. Similarly, if $\gamma_{2}<+\infty$, then (4.34) and formula (4.40) applied with $c=\gamma_{2}>\gamma_{1}$ imply that, for each $x \in \mathbb{R}$, the $\omega$-limit set of the function $t \mapsto u\left(t, \gamma_{2} t+x\right)$ is equal to the interval $\left[0, U_{\gamma_{2}}\left(x+x_{2}\right)\right]$.

Remark 4.2 The complex dynamics shown in Theorem 2.7 for the nonlinear equation (1.1) resembles that already known for the pure heat equation $\partial_{t} \zeta=\partial_{x x} \zeta$. Namely, there are initial conditions $\zeta_{0} \in L^{\infty}(\mathbb{R})$, which oscillate between $\operatorname{essinf}_{\mathbb{R}} \zeta_{0}$ and $\operatorname{esssup}_{\mathbb{R}} \zeta_{0}$ on larger and larger intervals, and for which the $\omega$-limit set of the function $t \mapsto \zeta(t, x)$ is equal to the whole interval $\left[\operatorname{essinf}_{\mathbb{R}} \zeta_{0}, \operatorname{esssup}_{\mathbb{R}} \zeta_{0}\right]$ for each $x \in \mathbb{R}$. This phenomenon was first pointed out by Collet and Eckmann [5]. Somehow, for the nonlinear equation (1.1), the complex dynamics appears when the initial condition $u_{0}$ oscillates on larger and larger intervals between two exponentially decaying functions with different decay rates. For such $u_{0}$, the proof of Theorem 2.7 shows that the solution $u$ oscillates between the two nonlinear travelling fronts whose speeds are different and associated to the two decay rates of $u_{0}$. Related complex behaviours have also 
been exhibited for reaction-diffusion equations in bounded domains [19], or in $\mathbb{R}^{N}[20]$ with power-like nonlinearities, where the $\omega$-limit set may be a continuum of equilibria. For (1.1) in $\mathbb{R}^{N}$ with $N \geq 2$ and bistable nonlinearities $f$ for which both 0 and 1 are stable, the $\omega$-limit set may be a continuum of translates of the same travelling front [21], the speed being unique in this case. Lastly, we refer to [10] for some recent convergence and non-convergence results to a unique or an interval of speeds of travelling fronts connecting 1 to any constant in $[0, \theta]$ for problem (1.1) with combustion-type nonlinearities $f$ vanishing on $[0, \theta]$ with $\theta \in(0,1)$.

Acknowledgements. The authors acknowledge the anonymous reviewers for their valuable comments on the manuscript.

\section{References}

[1] D.G. Aronson and H.F. Weinberger. Multidimensional nonlinear diffusions arising in population genetics, Adv. Math. 30 (1978), 33-76.

[2] M. Bages, P. Martinez, and J.-M. Roquejoffre. How travelling waves attract the solutions of KPP equations, Trans. Amer. Math. Soc., to appear.

[3] H. Bohr, Almost Periodic Functions, Chelsea Publishing Company, New York, NY, 1947.

[4] M. Bramson. Convergence of Solutions of the Kolmogorov Equation to Travelling Waves, Memoirs Amer. Math. Soc. 44, 1983.

[5] P. Collet and J.-P. Eckmann. Space-time behaviour in problems of hydrodynamic type: a case study, Nonlinearity 5 (1992), 1265-1302.

[6] U. Ebert and W. van Saarloos. Front propagation into unstable states: universal algebraic convergence towards uniformly translating pulled fronts, Physica D 146 (2000), 1-99.

[7] R.A. Fisher. The advance of advantageous genes. Ann. Eugenics 7 (1937), 335-369.

[8] K.P. Hadeler and F. Rothe. Travelling fronts in nonlinear diffusion equations, J. Math. Biol. 2 (1975), 251-263.

[9] F. Hamel and L. Roques. Fast propagation for KPP equations with slowly decaying initial conditions, J. Diff. Equations 249 (2010), 1726-1745.

[10] F. Hamel and Y. Sire. Reaction-diffusion spreading speeds for general initial conditions, SIAM J. Math. Anal. 42 (2010), 2872-2911.

[11] Y. Kametaka. On the nonlinear diffusion equation of Kolmogorov-Petrovski-Piskunov type, Osaka J. Math. 13 (1976), 11-66.

[12] A.N. Kolmogorov, I.G. Petrovsky, and N.S. Piskunov. Étude de l'équation de la diffusion avec croissance de la quantité de matière et son application à un problème biologique, Bull. Univ. État Moscou, Sér. Internat. A 1 (1937), 1-26. 
[13] D.A. Larson. Transient bounds and time-asymptotic behavior of solutions to nonlinear equations of Fisher type, SIAM J. Appl. Math. 34 (1978), 93-103.

[14] K.-S. Lau. On the nonlinear diffusion equation of Kolmogorov, Petrovsky, and Piscounov, J. Diff. Equations 59 (1985), 44-70.

[15] P. Martinez and J.-M. Roquejoffre. Convergence to critical waves in KPP-type equations, preprint.

[16] H.P. McKean. Application of Brownian motion to the equation of Kolmogorov-PetrovskiiPiskunov, Comm. Pure Appl. Math. 28 (1975), 323-331.

[17] H.J.K. Moet. A note on the asymptotic behaviour of solutions of the KPP equation, SIAM J. Math. Anal. 10 (1979), 728-732.

[18] W. Parry, Topics in Ergodic Theory, Cambridge Univ. Press, 1981.

[19] P. Poláčik and K.P. Rybakowski. Nonconvergent bounded trajectories in semilinear heat equations, J. Diff. Equations 124 (1996), 472-494.

[20] P. Poláčik and E. Yanagida. On bounded and unbounded global solutions of a supercritical semilinear heat equation, Math. Ann. 327 (2003), 745-771.

[21] J.-M. Roquejoffre and V. Roussier-Michon. Nontrivial large-time behaviour in bistable reaction-diffusion equations, Ann. Mat. Pura Appl. 188 (2009), 207-233.

[22] F. Rothe. Convergence to pushed fronts, Rocky Mountain J. Math. 11 (1981), 617-633.

[23] D.H. Sattinger. Stability of waves of nonlinear parabolic systems, Adv. Math. 22 (1976), 312-355.

[24] K. Uchiyama. The behavior of solutions of some semilinear diffusion equation for large time, J. Math. Kyoto Univ. 18 (1978), 453-508. 